\title{
Object-Based 3-D Reconstruction of Arterial Trees from Magnetic Resonance Angiograms
}

\author{
Jeffrey A. Fessler, Member, IEEE, and Albert Macovski, Fellow, IEEE
}

\begin{abstract}
This paper describes an object-based approach to the problem of reconstructing three-dimensional descriptions of arterial trees from a few angiographic projections. The method incorporates $a$ priori knowledge of the structure of branching arteries into a natural optimality criterion that encompasses the entire arterial tree. This global approach enables reconstruction from a few noisy projection images. We present an efficient optimization algorithm for object estimation, and demonstrate its performance on simulated, phantom, and in vivo magnetic resonance angiograms.
\end{abstract}

\section{INTRODUCTION}

$\mathrm{T}^{\mathrm{H}}$ HE inaccuracy of visual interpretation of angiograms has been well documented, and has motivated the development of automated methods for quantifying arterial morphology. Accurate descriptions of arterial trees would be useful for quantitative diagnosis of atherosclerosis, for surgical or treatment planning, for monitoring disease progress or remission, and for comparing efficacies of treatments [1]. This paper describes a new method for computing 3-D descriptions of arterial trees from a few projection images.

A summary of recent studies [2] discussed "a fascinating but disturbing fact $\cdots$ asymptomatic subjects with normal results on stress tests had a much lower mortality rate than asymptomatic subjects with abnormal results. However, the very large low-risk group with normal responses to exercise testing -.. contained, in absolute terms, the large majority of subjects who subsequently died suddenly." This observation highlights the importance of noninvasive quantitative angiography, since asymptomatic patients do not qualify for invasive examinations such as intra-arterial angiography. To identify individuals at possible risk due to mild plaque build-up, a noninvasive procedure is needed that can accurately quantify lumen size. Magnetic resonance angiography (MRA), in conjunction with computed reconstruction of arterial trees, has potential to serve this need. The 3-D arterial reconstruction algorithm presented in this paper is particularly suited to MR data, though the approach is also applicable to digital X-ray angiograms.

The projection model analyzed in this paper is based on the selective inversion-recovery (SIR) method [3] for MRA. "SIR bears some similarities to X-ray digital subtraction angiography (DSA). While DSA involves the subtraction of two images, one with and the other without contrast agent, SIR involves the sub-

Manuscript received March 5, 1990; revised July 16, 1990. This work was supported in part by the National Institute of Health under Grants HL39297, HL-34962, and HL-39478, the National Science Foundation under Contract ECS-8213959, and GE Medical Systems Group under Contract $22-84$

J. A. Fessler is with the Division of Nuclear Medicine, University of Michigan Medical Center, Ann Arbor, MI 48109-0552.

A. Macovski is with the Department of Electrical Engineering, Information Systems Laboratory, Stanford University, Stanford, CA 95305.

IEEE Log Number 9041388 traction of two images, one with and the other without inversion excitation of blood prior to its entering the region of interest." [4] By using a multiple-readout selective inversion-recovery (MRSIR) sequence [5], we can acquire arterial views at a few projection angles without using invasive contrast agents or ionizing radiation. A typical MRSIR sequence with 256 cardiacgated phase-encode pairs lasts about $4 \mathrm{~min}$. During each cardiac cycle, several spatial-frequency space readouts occur-each corresponding to a difficult projection angle. For a multiplereadout sequence, if the number of readouts is increased, then the tip-angles must be decreased. Thus, the signal-to-noise ratio per view is inversely related to the number of projection angles, which necessitates optimal use of the projection data. (Flowsensitive MR techniques can acquire a 3-D data set directly [6], [7], but the large number of required excitations makes cardiac gating impractical. Ungated sequences often suffer from sensitivity to non-uniform flow and vessel motion.)

Projection-reconstruction from only a few views in an illconditioned problem in general, but as observed by Rossi [8], "the ultimate goal of processing the projection measurements is typically far more modest than obtaining high resolution cross-sectional imagery." In fact, the goal is typically to obtain quantitative descriptions of arterial shape (perhaps as an intermediate step towards the goal of evaluating hemodynamic properties). Therefore, rather than attempting to compute 3-D voxels, we use an elliptical model to approximate arterial crosssections. This parametric approach exploits our a priori knowledge of the structure of arteries, and translates the reconstruction problem into an object estimation problem. However, a single ellipse is inadequate for representing bifurcations where stenoses frequently occur. This paper describes a new extension of the generalized-cylinder object model to overcome this limitation, allowing the first application of our method to projection data acquired in vivo.

The novel aspects of the method we present are as follows. It is based on a global criterion-to maintain accuracy at the low SNR typical of noninvasive methods; bifurcating arteries are explicitly modeled; there are no empirically determined thresholds; overlapping vessel projections are accommodated; and the time-dependence of contrast density is modeled. The theory we present has the potential of providing a fully automatic reconstruction algorithm. However, like many methods, the current implementation of our algorithm requires some manual initialization. Automating this will be a necessary step towards making the algorithm clinically useful.

The paper is organized as follows. In Section II, we briefly review other 3-D reconstruction methods. In Section III, we present a statistical model relating the projection images to the unknown arterial tree. In Section IV, we describe a new object model, tailored for representing arterial trees. In Section V, we propose a new optimality criterion for the problem of estimating 
an arterial tree from noisy projections. This criterion is optimized using the estimation algorithm presented in Section VI. In Section VII, we show the results of applying this algorithm to simulated angiograms, to MR phantom data, and to MRA projections of carotid arteries. We discuss the results and the future directions of this research in Section VIII.

\section{BACKGROUND}

Quantitative angiography has been studied for over two decades, and improvements in imaging technology and computer speed continue to spur the development of various reconstruction approaches for the many different applications. In this section, we review some of these methods; see also [9], [10] for more comprehensive reviews. To our knowledge, all previous methods have been applied only to X-ray angiograms and have not addressed the unique aspects of MRA.

An arterial tree can be described with the most generality by a three-dimensional density function $\mu(x, y, z ; t)$. In general, $\mu$ is also a function of time due to the motion of blood and of the arteries. In X-ray imaging, $\mu$ represents the linear attenuation coefficient of iodinated blood, and $\mu$ is a real, positive quantity. In magnetic resonance angiography (MRA), $\mu$ represents the density of inverted spins that have flowed into the readout region [3], [5], and in general is a complex quantity due to spin dephasing. This aspect is discussed further in the next section. We use "contrast" or "density" throughout to refer to $\mu$, although no contrast agent is used in MRA. Though specialized X-ray equipment [11], [12] can acquire enough projection data to reconstruct a discretized estimate of $\mu$, the timevariation of arteries limits acquisition to only a few (almost) simultaneous projection images with practical equipment. For some applications, estimates of arterial position and area may be sufficient, and algorithms have been published for obtaining those features from just two views [9], [13], [14]. However, computations of hemodynamic significance depend on stenosis geometry, including entrance and exit angle [15], for which area alone is insufficient. To circumvent the ill-conditioned nature of limited-view reconstruction, virtually all methods attempt to reduce the dimensionality by considering some special class of densities.

Perhaps the most flexible approach is to consider $\mu$ to be a discretized 3-D voxel set, and to incorporate a priori knowledge such as sparseness, non-negativity, and connectedness to regularize the reconstruction [16], [17]. Though this method has the ability to (discretely) represent any arterial shape, accurate reconstruction seems to require several views. To reduce the number of views needed, $\mu$ is often restricted to binary values indicating the presence or absence of blood in each voxel. This implicitly assumes that the contrast uniformly fills the entire arterial tree, and that the contrast density is known. These assumptions are violated in MRA, due to variable "wash-in." Other methods assume that only a single artery is imaged, or that the cross-sections are ellipses with axes aligned with two orthogonal projection angles. Often the restrictions on $\mu$ are only made implicitly, such as when "diameter" is computed from a single view.

To compensate for limited views and low SNR, stronger assumptions are necessary. The model-based approach of this paper is rooted in the work of Rossi [8], who analyzed reconstruction of a circular object from projections, and of Shmueli [18], who developed a dynamic-programming algorithm for outlining a single cylindrical artery in one view. Pappas [19] demon- strated the accuracy of using elliptical cross-sections to represent arteries, and Bresler [20] presented an optimal algorithm for reconstructing a single object with elliptical cross-sections from a few projections. In [21], Rossi further evaluated ellipse reconstruction accuracy. Kitamura et al. [9] presented an algorithm for computing the skeleton and transverse areas of an arterial network from a single view, assumed to be free of overlapping arteries, and demonstrated 3-D reconstruction of a single artery from biplane angiograms. Their approach was also based on the generalized-cylinder object model, as was our work with Bresler [22], [23]

An ellipse can better approximate a wider class of cross-sections than can a circle, but has the disadvantage that more than two views are required to obtain a unique reconstruction. Spears [24] has calculated the deterministic error in reconstructing ellipses from just two views. For applications where very accurate quantification of stenosis geometry is critical, one can justify acquiring more views. Nevertheless, to maximize SNR with the MRSIR method, we would like to use as few views as is possible. Bresler [25] proved that an arbitrary set of ellipses can be reconstructed (with probability one) from only three (ideal) views. In the presence of measurement error, discretization, and blurring, we conjecture that four views is the minimum plausible number.

With the exceptions of [18], [20], most of the model-based $3-\mathrm{D}$ reconstruction algorithms and many of the 2-D arterial tracking procedures have consisted of two steps: estimation of cross-sectional features on a slice-by-slice basis, followed by some sort of smoothing of those features [9], [15], [26]-[28]. The smoothing filter is usually chosen heuristically, with an empirically determined bandwidth. These two-step approaches are suboptimal, even if each step is optimal [22], but how much so is unclear. By using ellipses, we capture our a priori knowledge about the shape of arterial cross-sections in a simple parametric form. The equally important a priori knowledge that arteries are smooth, i.e., ellipse parameters vary slowly along an arterial segment, is more difficult to quantify. Shmueli [18] and Bresler [20] used parametric Gauss-Markov models to quantify this smoothness parametrically. We have two objections to this approach: 1) the parameters of a Gauss-Markov model are unknown and not easily determined, and 2) in general, these models imply that the a priori covariances of the cross-sections vary along the length of an artery. This implied variation is inconsistent with our intuition: prior to examining an angiogram, our uncertainty about cross-sectional shape is uniform along the arteries. We instead propose a nonparametric approach, described in Section $\mathrm{V}$, that captures our a priori knowledge of arterial smoothness with minimal assumptions.

\section{Measurement Model}

Reconstruction from projections belongs to the mathematical class called inverse problems. Solutions to such problems depend heavily on the assumptions one makes about the corresponding forward processes. In this section, we present a statistical model for projection angiography that relates an unknown arterial tree to its acquired measurements. This model accounts for overlapping arterial projections, space-invariant blurring, and additive noise. In the next section, we will restrict our attention to a specific class of arterial tree descriptions, but for this section, we represent an arterial tree by an arbitrary three-dimensional density function. DSA images may also be corrupted by an unknown background field, and one could ex- 
tend the model to account for an unknown background [9], [19], [29], [30]. This has not been necessary for MRA images.

The density function for an arterial tree varies with time, and a reconstruction paradigm that requires multiple views must account for this variation. Since the MRSIR readouts occur during diastole, we assume that the arteries' positions are identical for each projection angle. Since each readout takes only $6 \mathrm{~ms}$, "wash-in" effects during the readout are negligible. However, the contrast may still change between readouts, and we account for this variation in our measurement model. We assume $P$ projection images are acquired at time instants ${ }^{1} t_{1}, \cdots, t_{P}$ and at projection angles $\theta_{1}, \cdots, \theta_{P}$. For example, for a dual-biplane DSA system [29]: $P=4, t_{1}=t_{2}, t_{3}=t_{4}, \theta_{1}=0^{\circ}, \theta_{2}=90^{\circ}$, $\theta_{3}=\Delta_{\theta}$, and $\theta_{4}=\Delta_{\theta}+90^{\circ}$.

A projection is a mapping of a 3-D density into a 2-D image, and that mapping is determined by the acquisition geometry. MR projection images emulate a parallel geometry, for which the projection mapping is expressed as line-integrals through the density [31]

$l_{\theta_{p}}(u, v ; \mu)=$

$$
\int \mu\left(s \cos \theta_{p}+u \sin \theta_{p}, s \sin \theta_{p}-u \cos \theta_{p}, v ; t_{p}\right) d s
$$

where $\mu(x, y, z ; t)$ is the time-varying 3-D density and $l_{\theta}(u, v$; $\mu$ ) is the projection of $\mu$ at angle $\theta$. As shown in Fig. 1, $u$ and $v$ are the local coordinates in the projection images, and the $z$-axis is the "rotational axis." (The $z$ and $v$ coordinates are equivalent.) The density function represents only the contribution of (lumens of) the arterial tree of interest, assuming any other tissues are removed by subtraction. The restriction to a geometry with a single axis of rotation is convenient for reconstruction, but a more sophisticated approach would utilize the ability of MR systems to acquire projections in any direction.

As mentioned above, the density $\mu$ is in general a complex quantity, as are the projection images. Unfortunately, the complex image data is typically corrupted by spurious phase, so we use a homodyning procedure [32], [33] to extract the desired in-phase component of the images. If $\mu$ has significant dephasing, then there may be signal cancellation that is not modeled by (1). Our approach has been to minimize dephasing by using very short readouts (partial $k$-space) [4], by selectively projecting only a slab containing the branches of interest [34], and by carefully shimming the main field [35]. The model used here (1) should be generalized for systems with poor homogeneity.

Actual imaging systems produce noisy, discrete samples of a blurred version of the ideal projections, and accurate estimation requires accounting for these distortions. In MR, the pointspread function (PSF) is determined by the frequency-space sampling and weighting, and is spatially invariant. Although it is possible to estimate the point-spread function and the arterial tree [9], [19] simultaneously, Pappas [19] has observed that even "if we assumed that there is no blurring, we would still get good estimates of the parameters." Here, we assume the PSF is known, spatially invariant, and denoted by $h(u, v)$.

Digital imaging systems acquire samples of the blurred projections in the form of images. Let these images consist of $N$ rows of $W$ pixels each. If $s_{\theta_{r, \ldots, i}}(\mu)$ denotes the ideal mean $i$ th pixel value in the $n$th row of the projection image at angle $\theta_{p}$,

'These times are relative to the cardiac $R$-wave.

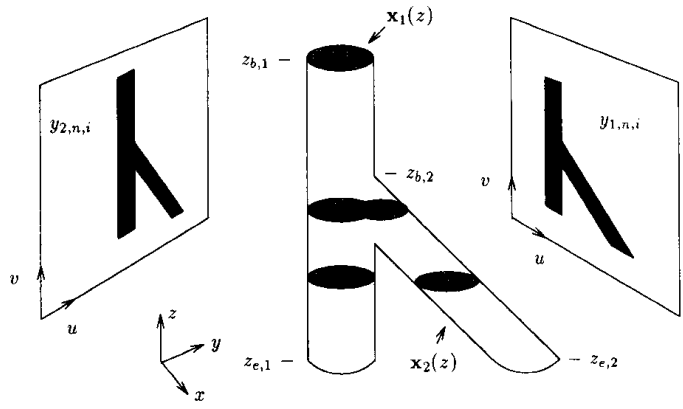

Fig. 1. Projection geometry, showing projections of a bifurcation.

then for $i=1, \cdots, W ; n=1, \cdots, N$; and $p=1, \cdots, P$ :

$$
\left.s_{\theta_{p, n, i}}(\mu) \triangleq\left[h(u, v) * * l_{\theta_{i}}(u, v ; \mu)\right]\right|_{u=\left(i-i_{h}-(1 / 2)\right) \Delta_{i, l^{\prime}=z_{u}}}
$$

where $z_{n} \triangleq(N-n) \Delta_{l}$, the horizontal and vertical pixel dimensions are $\Delta_{h}$ and $\Delta_{r}$, respectively, $i_{h}$ is the distance (in pixels) from the projection of the rotation axis to the left edge of a projection image, and $* *$ denotes the $2-\mathrm{D}$ convolution operator. The offset $i_{h}$ may differ from its usual value of $W / 2$ since any extra linear phase in an MR system leads to a shift in the reconstructed images.

Blurring along the length of an artery's projection is less important for reconstruction than lateral blurring that smooths the artery's edges. In the next section we will restrict our attention to arteries whose medial axes are roughly parallel to the $z$ axis, so it is reasonable to ignore blurring along this axis. This approximation simplifies the estimation algorithm discussed in Section VI. Furthermore, if we assume that the PSF is smooth, then we can approximate (2) by a discrete convolution that is more easily implemented. These approximations allow us to rewrite (2) as

$$
s_{\theta, n, i, j}(\mu) \approx h_{i} * l_{\theta_{p, i}}\left(z_{n,} ; \mu\right)
$$

where * now indicates discrete convolution with respect to $i$, and

$$
\begin{aligned}
h_{i} & \triangleq h\left(i \Delta_{h}\right), \\
l_{\theta_{p, i}}(v ; \mu) & \triangleq \int_{(i-1) \Delta_{h}}^{i \Delta_{h}} l_{\theta_{p}}\left(u-i_{h} \Delta_{h}, v ; \mu\right) d u .
\end{aligned}
$$

Finally, the actual measured pixel values are corrupted by noise. If $y_{p, n, i}$ denotes the measurement corresponding to $s_{\theta_{p, \mu, i}}$, then we assume

$$
y_{p, n, i}=s_{\theta_{p, n, i}}(\mu)+\epsilon_{\theta_{p, n i}}
$$

where the additive measurement error $\epsilon_{p, n, i}$ has a Gaussian distribution with (possibly unknown) variance $\sigma^{2}$. In MR imaging, the "source of this noise is thermally generated, randomly fluctuating noise currents in the body which are picked up by the receiving antenna," [36] so it is correct to assume that the measurement errors are Gaussian and independent.

It is convenient to group the ideal projection and measurement samples in the different ways defined below. First, group the samples by rows

$$
\begin{aligned}
y_{p, n} & \triangleq\left[y_{p, n, 1}, \cdots, y_{p, n, w}\right]^{\prime}, \\
s_{\theta_{p, n}}(\mu) & \triangleq\left[s_{\theta_{p, n, 1}}(\mu), \cdots, s_{\theta_{p, n, w}}(\mu)\right]^{\prime}
\end{aligned}
$$


where "' "' denotes matrix transposition. Next, group the corresponding rows of the $P$ images

$$
\begin{aligned}
\boldsymbol{y}_{n} & \triangleq\left[\boldsymbol{y}_{1, n}^{\prime}, \cdots, \boldsymbol{y}_{P, n}^{\prime}\right]^{\prime}, \\
s_{n}(\mu) & \triangleq\left[s_{\theta_{1, n}}(\mu)^{\prime}, \cdots, s_{\theta P, n}(\mu)^{\prime}\right]^{\prime} .
\end{aligned}
$$

Finally, let

$$
y \triangleq\left[y_{1}^{\prime}, \cdots, y_{N}^{\prime}\right]^{\prime}, s(\mu) \triangleq\left[s_{1}(\mu)^{\prime}, \cdots, s_{N}(\mu)^{\prime}\right]^{\prime}
$$

be the aggregates of all the samples. The vector $y$ has length $N P W$. We similarly define $\epsilon$, yielding the final measurement model

$$
\boldsymbol{y}=s(\mu)+\epsilon
$$

which simply says that the (known) measurements are equal to the sampled and blurred projection of the (unknown) arterial tree density plus noise.

In summary, we have defined a statistical measurement model

(5), using the following assumptions:

- negligible dephasing,

- linear projection of density function,

- uniform background (or presubtracted background),

- known, space-invariant PSF,

- parallel geometry, and

- independent, additive, Gaussian measurement errors.

\section{OBJECt MODEL}

As discussed in the introduction, the information provided by only a few projections is insufficient for reconstructing a general three-dimensional function. Any solution therefore hinges on restricting consideration to a smaller class of descriptions. In this section, we introduce an extension of the generalized cylinder (GC) object model, tailored to the task of representing arterial trees (the terms "object" and "arterial segment" are interchangeable). In particular, our model is designed to represent bifurcations accurately. This accuracy is essential since atherosclerotic lesions are prevalent near arterial branches [37], [38]. Binford [39], [40] introduced the GC model for computer vision applications. According to Agin [40], "a generalized cylinder consists of a space curve, or axis, and a cross-section function defined on that axis." For this paper, we take that cross-section function to be an ellipse.

\section{A. SGC Object Model}

A true GC would have arbitrarily oriented ellipses, as in Fig. 2. Reconstruction of such general objects from projections is still an open problem; to our knowledge, the only work on this problem is a hierarchical algorithm proposed, but never implemented, by Bresler [25]. The comments of Marr [41, pp. 223224] suggest that GC reconstruction may be challenging to implement from projections acquired around a single rotation axis. We instead adopt the popular approach [9], [20], e.g., of parameterizing objects by their cross-sections parallel to the $x y$ plane, an approach ideally suited to the cylindrical geometry. As argued in [9], a GC with slowly varying elliptical crosssections can be approximated by a set of parallel ellipses as shown in Fig. 2. Such a set of ellipses can be parameterized by $z$, hence we call the collection a single-valued generalized cylinder (SGC). Objects that wind back upon themselves (e.g., $U$ shaped) must be represented by more than one SGC, and are

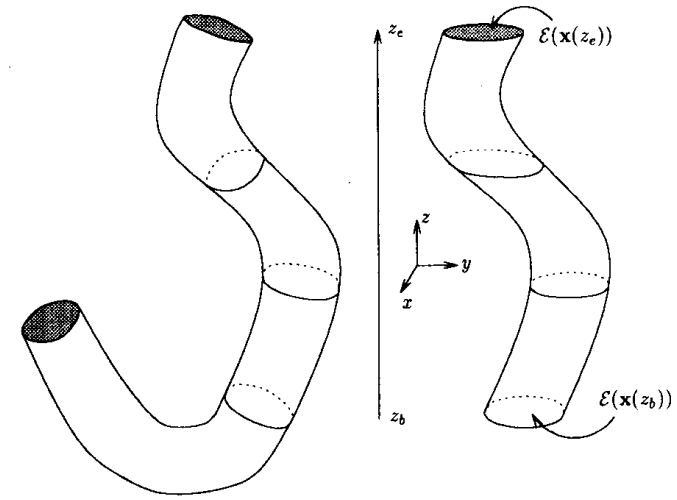

Fig. 2. A GC (left) and an approximating SGC (right).

called "multivalued." The further an object is tilted away from the rotational axis, the poorer its SGC representation. Thus, as in most imaging procedures, proper patient positioning is essential, and the arteries of interest should be aligned as close as possible to the rotational axis. The examples of Section VII demonstrate successful reconstruction of objects with tilts exceeding $45^{\circ}$. Though we parameterize objects by parallel crosssections, the cross-sections perpendicular to an artery's medial axis are more important for quantitative diagnosis. Hence, we present formulae for converting between GC and SGC parameters in Appendix C.

Each SGC cross-section has three attributes: position, shape, and content. An ellipse's position attribute is parameterized by the $x y$ coordinates of its center, denoted by $\left(c_{x}, c_{y}\right)$. We parameterize an ellipse's shape attribute by its radius (geometric mean of long and short axes), eccentricity ${ }^{2}$ (ratio of long to short axis) ${ }^{3}$, and orientation, denoted by $r, \lambda$, and $\phi$, respectively (see Fig. 3). The content attribute parameterizes the density within an artery, and can have a profound, though often underestimated, impact on a reconstruction algorithm's accuracy and applicability. Some methods assume the content is known or is computable from "normal" sections of an artery, usually assumed to have a circular cross-section. Having more than two views allows us to relax these assumptions. We do not assume the contrast density is known, and we allow it to vary along the length of an artery, from artery to artery, and possibly even between projection images, as discussed next.

Though the measurement model (5) allows a general timevarying density, we now consider only SGC's whose position and shape attributes are invariant at times $t_{1}, \cdots, t_{P}$, but we account for the time variation of the content attribute. ${ }^{4}$ This approach is necessary since the contrast often varies between MRA projection images, and is approximately sufficient since cardiac gating can synchronize position and shape. We assume each particular arterial cross-section is uniformly filled with contrast agent, so we parameterize an ellipse's content by a vector denoted by $\boldsymbol{\rho}=\left[\rho_{1}, \cdots, \rho_{P}\right]^{\prime}$ where $P$ is the number of projections. For some imaging techniques, we may be able to

${ }^{2}$ The radius/eccentricity parameterization is preferable to the long-axis/ short-axis parameterization for our approach, since independence of radius and eccentricity is a more realistic assumption, particularly in stenotic arteries.

${ }^{3}$ Another definition of ellipse eccentricity is $\sqrt{1-\lambda^{-2}}$. [29].

${ }^{4} \mathrm{We}$ could also account for lateral vessel motion between acquisitions 


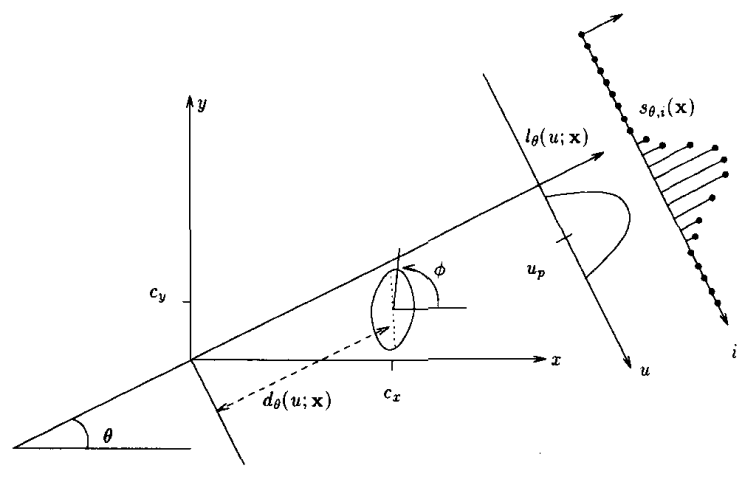

Fig. 3. Predictions of an elliptical cross-section. $l_{\theta}$ : ideal, $s_{\theta, i}$ : blurred and sampled.

equate some of the $\rho_{\rho}$ 's. For example, with biplane DSA, the contrast is identical for each pair of projection images, so we would assume $\rho_{1}=\rho_{2}$ and $\rho_{3}=\rho_{4}$. Note that ideal data sets would have $\rho_{1}=\cdots=\rho_{P}$, since estimating additional parameters often decreases estimation accuracy, but we have found the full generality presented here necessary for some MRA data.

We now define notation for a hierarchy consisting of ellipses, objects, and trees. The symbol $x$ will denote a particular set of $5+P$ ellipse parameters. In particular,

$$
\boldsymbol{x}_{k}(z)=\left[\begin{array}{c}
c_{x}(z) \\
c_{y}(z) \\
r(z) \\
\lambda(z) \\
\phi(z) \\
\rho(z)
\end{array}\right]
$$

denotes the (unknown) ellipse parameters of the $k$ th object at vertical position $z$. An object is uniquely determined by specifying its starting plane $z_{b}$, its ending plane $z_{e}$, and the collection of parameters of its elliptical cross-sections between those planes. We use the symbol $\mathcal{O}_{k}$ to denote the $k$ th object, i.e.,

$$
\mathcal{O}_{k} \triangleq\left\{z_{k}, x_{k}\right\}
$$

where

$$
z_{k} \triangleq\left[z_{b, k}, z_{e, k}\right]
$$

denotes the vertical domain of the $k$ th object, and

$$
x_{k} \triangleq\left\{x_{k}(z): z \in \mathbb{Z}_{k}\right\}
$$

denotes the collection of ellipse parameters. We will say more about $Z_{k}$ later in this section.

An arterial tree is a collection of $K$ objects, or "object-set," denoted by the symbol $\Psi$

$$
\begin{aligned}
\Psi & \triangleq\left\{K, \mathcal{O}_{1}, \cdots, \mathcal{O}_{K}\right\} \\
& =\left\{K, \mathcal{z}_{1}, x_{1}, \cdots, \mathcal{Z}_{K}, x_{K}\right\} .
\end{aligned}
$$

The notational hierarchy for $\boldsymbol{\Psi}$ directly corresponds to a hierarchical data structure [13] that we used to implement the algorithm described in Section VI. Due to the simplicity of the ellipse parameterization, this representation for $\boldsymbol{\Psi}$ is considerably more compact than a discretized 3-D voxel set. More im- portantly, the significant factors for quantitative diagnosis, such as percent stenosis and stenosis eccentricity, are directly computable from $\boldsymbol{\Psi}$.

With these definitions, we have translated the problem of reconstructing a density $\mu$ from projections into the problem of estimating an object-set $\boldsymbol{\Psi}$ from projections. That is, we must estimate the number of objects, the vertical extent of each object, and the parameters of the ellipse cross-sections for each object.

\section{B. Branching Arteries Model}

In earlier efforts [22], [23], we assumed that the $K$ objects to be reconstructed were disjoint. Although convenient, this assumption precluded accurate modeling of branching vessels. Histological sections of bifurcations [37], [38] suggest that pairs of intersecting ellipses can approximate cross-sections of a bifurcation accurately, even a diseased one. Therefore, we discard the disjointedness assumption by allowing SGC objects to intersect and by accounting ${ }^{5}$ for their intersections [42]. The importance of this accounting is clear from early GC-based vision algorithms [40] that were prone to failure near the intersection of GC's. (For simplicity, we consider bifurcations only; the extension to trifurcations involves only additional notation.)

When two SGC objects intersect, their position and shape attributes are unaffected, but we must specify their content attributes more carefully. Although it may be reasonable to assume that two intersecting ellipses must share the same density, we can simplify our reconstruction algorithm by not enforcing this constraint. This also provides a self-test for the reconstruction: if the estimated densities differ significantly for two arterial branches near a bifurcation, then something is clearly amiss. For mathematical consistency, we must specify a symmetrical definition for the content of two intersecting ellipses with different densities. Our convention is described pictorially in Fig. 4 where we define the density of the common area of two ellipses to be the average of the two ellipse densities. If the two densities are the same, then the area of overlap will have that same density.

With the above definitions, we can now specify the unique 3-D density function generated by a given object-set. Let $\mathcal{E}(\boldsymbol{x})$ be the set of points within the ellipse parameterized by $x$, i.e., if $\boldsymbol{x}=\left[\begin{array}{lll}c_{x} & c_{y} & r \lambda \phi \boldsymbol{\rho}^{\prime}\end{array}\right]^{\prime}$, then

$$
\begin{aligned}
\mathcal{E}(\boldsymbol{x})= & \left\{(x, y):\left(\frac{\left(x-c_{x}\right) \cos \phi-\left(y-c_{y}\right) \sin \phi}{r \sqrt{\lambda}}\right)^{2}\right. \\
& \left.+\left(\frac{\left(x-c_{x}\right) \sin \phi+\left(y-c_{y}\right) \cos \phi}{r / \sqrt{\lambda}}\right)^{2} \leq 1\right\} .
\end{aligned}
$$

The relationship between an object-set $\boldsymbol{\Psi}$ and its density $\mu_{\boldsymbol{\Psi}}$ is then

$$
\begin{aligned}
& \mu_{\mathbf{\Psi}}\left(x, y, z ; t_{p}\right)= \\
& \sum_{k=1}^{K}\left[\rho_{k, p}(z) 1_{\mathcal{E}\left(x_{k+i}\right)}(x, y) 1_{z_{k}}(z)\right] \\
& -\sum_{k_{1}=1}^{K} \sum_{k_{2}=1}^{K}\left[\frac{\rho_{k_{1}, p}(z)+\rho_{k_{2}, p}(z)}{2}\right. \\
& \left.\cdot 1_{\mathcal{E}\left(x_{k_{1}}(z)\right)}(x, y) 1_{\varepsilon\left(x_{k_{2}}(z)\right)}(x, y) 1_{z_{k_{1}}}(z) 1_{z_{k_{2}}}(z)\right] \text {, }
\end{aligned}
$$

${ }^{5}$ More perspiration than inspiration: compare Appendices $\mathbf{A}$ and $\mathbf{B}$. 


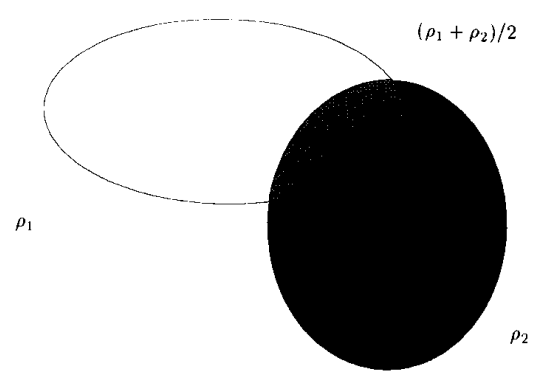

Fig. 4. Convention defining content attribute of a cross-section formed by two intersecting ellipses with different densities.

where

$$
1_{z}(z)=\left\{\begin{array}{ll}
1, & z \in \mathbb{Z} \\
0, & z \notin \mathbb{Z}
\end{array} .\right.
$$

The first summation would suffice for a set of disjoint objects, and the second summation accounts for the areas of overlap.

The reverse relationship to $(8)$ is not unique because many different object-sets generate the same density function. One trivial reason for this nonuniqueness is that an ellipse's shape is the same for orientations $\phi$ and $\phi+\pi$, but the parameter vectors differ. Also, if one changed $\Psi$ by adding objects with zero density, there would be no change in $\mu_{\Psi}$. More significantly, the union of two identical ellipses is indistinguishable from a single ellipse. We will say two object-sets are equivalent if the density functions they generate from (8) are equal. For example, if the three objects shown in Fig. 5 all have the same density, then the object-set formed by combining $\mathcal{O}_{1}$ and $\mathcal{O}_{2}$ is equivalent to the object-set formed by combining $\mathcal{O}_{1}$ and $\mathcal{O}_{3}$. Technically then, we will be reconstructing an equivalence class of object-sets from projections.

Since a given object-set $\boldsymbol{\Psi}$ generates a unique density function $\mu_{\Psi}$ by (8), we can speak of the projection of an object-set, denoted by $s\left(\mu_{\Psi}\right)$. Although $s$ is linear in $\mu$, it is not linear in $\boldsymbol{\Psi}$ since $\mu$ is a nonlinear function of $\boldsymbol{\Psi}$. In fact, since we are not assuming disjoint objects, $s$ is not even additive in general, i.e., the projection of an object collection differs from the sum of each object's projection. Nevertheless, we can write expressions for $s\left(\mu_{\Psi}\right)$ in closed form using the formulas derived in the appendices.

The nonuniqueness of an object-set actually works to our advantage since we need not estimate $z_{b}$ or $z_{c}$ exactly for objects near bifurcations, as demonstrated by Fig. 5 . What defines the endpoints of an arterial segment? There are four possibilities for SGC objects:

- an artery may leave the region of interest (ROI),

- an artery may "fade-out" due to incomplete filling by contrast agent,

- an artery may be occluded or taper down to a size below the effective resolution,

- or an artery may connect to another artery (branching).

These four possibilities are illustrated in Fig. 6. Of these, only the first possibility has a unique value of $z_{b}$ ( or $z_{e}$ ), and this value is easily determined. For the others, we can always conservatively make the object extra long, and let the density or radius become vanishingly small. For a multivalued GC object, the endpoints of its SGC approximation are poorly defined, and are determined in practice by the manual-entry described in Section VI.

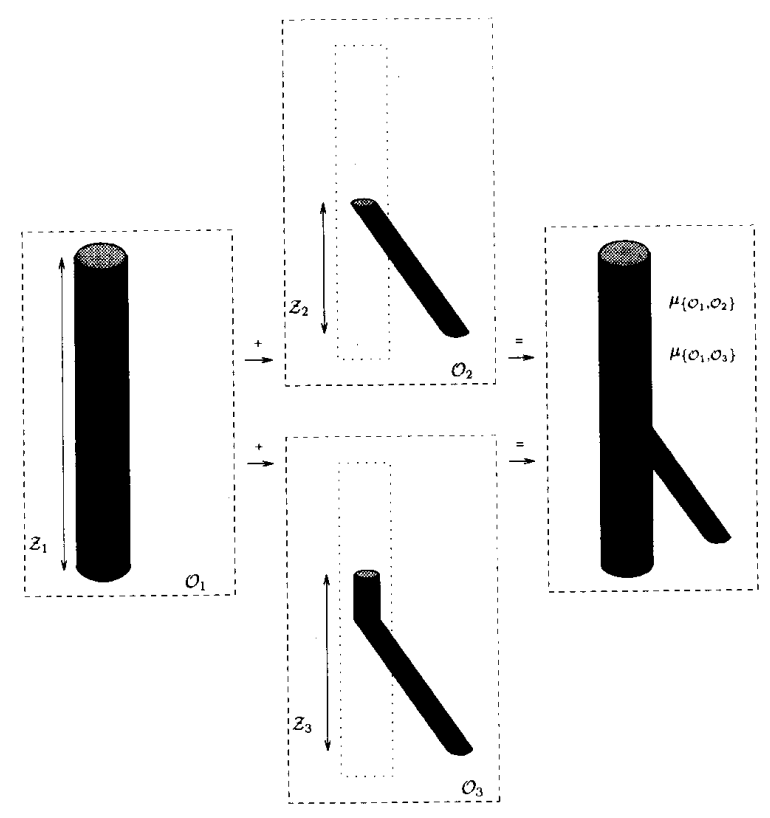

Fig. 5. Two equivalent object sets. Note that although $\dot{z}_{2} \neq z_{3}$, $\mu_{\left\{0_{1}, 0_{2}\right\}}=\mu_{\left\{0_{1}, 0_{3}\right\}}$.

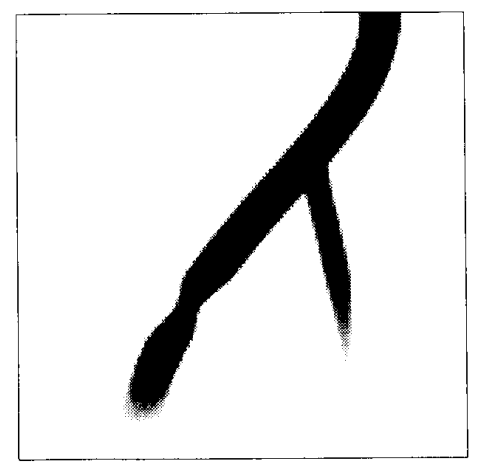

Fig. 6. Example demonstrating the four ways arteries can terminate

We have described an extension of the generalized-cylinder object model based on elliptical cross-sections. By allowing objects to intersect, the model can represent arterial trees accurately. This model defines the class of 3-D density functions considered, namely, those that can be expressed in the form (8).

\section{OBject Estimation}

Equipped with the measurement and object models of the previous sections, we can define criteria for the problem of estimating an unknown object-set $\boldsymbol{\Psi}$ from noisy projection measurements $y$. After presenting the maximum-likelihood criterion and noting its shortcomings, we introduce a nonparametric optimality criterion. Our approach is motivated by the success of nonparametric regression, especially cubic-spline smoothing, at estimating smooth curves from noisy data [43].

\section{A. Maximum Likelihood Criterion}

An obvious approach would be to find the object-set whose computed projections are the closest to the measurements in 
some sense. Under the independent Gaussian noise model, the least-squares estimate is also the maximum-likelihood (ML) estimate, defined by [cf. (6)]:

$$
\hat{\Psi}_{\mathrm{ML}} \triangleq \arg \min _{K} \min _{z_{1} \cdots, z_{K}} \min _{x_{1}, \cdots, x_{\kappa}}\left\|\boldsymbol{y}-s\left(\mu_{\Psi}\right)\right\|^{2} .
$$

This estimator is severely under-regularized; one can always add tiny objects whose parameters fit some bit of noise, thereby increasing the "likelihood," but certainly not improving the estimate. Even if the number of objects is fixed so that $K$ cannot grow without bound, the results will be unsatisfactory due to noise and the "threshold effect" of ML estimation [44], if the SNR is low. Indeed, many methods follow the ML estimation with a post-processing smoothing step.

The principal shortcoming of ML estimation is that it ignores our a priori knowledge of arterial smoothness. The nonparametric optimality criterion presented below attempts to capture this knowledge realistically.

\section{B. Nonparametric Optimality Criterion}

Smoothing always involves a tradeoff between the conflicting goals of fit to the measurements and smoothness of the estimated functions. Nonparametric regression offers a solution to this tradeoff, and only requires an assumption of integrability of the square of the second derivative of the estimand. Most nonparametric regression literature has been concerned with estimating scalar functions from linear, scalar measurements. The natural generalization of these "penalized-likelihood" [45] or "regularized" [46] methods to our nonlinear, multidimensional, object estimation problem is the following estimator and optimality criterion: ${ }^{6}$

$$
\begin{aligned}
\hat{\boldsymbol{\Psi}} & \triangleq \arg \min _{K} \min _{z_{1}, \cdots, z_{K} \boldsymbol{x}_{\mathbf{1}}(\cdot), \cdots, \boldsymbol{x}_{K}(\cdot)} \boldsymbol{\Phi}(\boldsymbol{\Psi}), \\
\boldsymbol{\Phi}(\boldsymbol{\Psi}) & \triangleq\left\|\boldsymbol{y}-\boldsymbol{s}\left(\mu_{\boldsymbol{\Psi}}\right)\right\|^{2}+\boldsymbol{\alpha}^{\prime} \sum_{k=1}^{K} \int_{z_{k}} \ddot{\boldsymbol{x}}_{k}^{2}(z) d z
\end{aligned}
$$

where $\ddot{x}(z)$ is the $(5+P)$-dimensional vector containing the second partial derivatives of the components of $x(z)$ with respect to $z$.

The smoothing tradeoff is controlled by the smoothing factor $\boldsymbol{\alpha}=\left[\alpha_{1}, \cdots, \alpha_{5+P}\right]^{\prime}$; for small $\boldsymbol{\alpha}$, the estimate fits the data closely (approaching the ML estimate as $\boldsymbol{\alpha}$ goes to zero), and for large $\boldsymbol{a}$, the estimate becomes very smooth. Intuitively, we would use the same fairly large value for $\alpha_{1}$ and $\alpha_{2}$ since ellipse position varies relatively slowly, very large identical values for $\alpha_{6}, \cdots, \alpha_{5+P}$, since density typically varies very slowly, and smaller values for $\alpha_{3}, \alpha_{4}$, and $\alpha_{5}$, to avoid oversmoothing the important shape features. These qualitative staements are unsatisfying theoretically. Fortunately, nonparametric regression offers a solution: we can estimate $\boldsymbol{\alpha}$ from the data itself using cross-validation [47]-[49]. We conjecture that arterial disease will primarily effect $\alpha_{3}$ and $\alpha_{4}$, so that the other elements of $\alpha$ could be fixed for a given imaging technique.

Why this criterion? The first term of (9) is the measurement negative-log-likelihood, which we would like to be small, but not at the expense of excessive object wiggliness. Functions

${ }^{6}$ This criterion is nonparametric in the sense that we have avoided using a parametric (e.g., Gauss-Markov) model for the evolution of cross-section parameters along an artery. that minimize, subject to specified constraints, the second term turn out to be splines, the smoothest functions (in curvature sense) satisfying those constraints. We argue that for maximum effectiveness, arteries attempt to be as smooth as possible, subject to anatomic constraints. An example is carotid arteries, which make very smooth trajectories from their origins at the aorta to their destinations in the head. These arguments are heuristic, and the reader may disagree; we hope this discussion prompts proposals of other criteria. Our main point here is that (9) is a natural optimality criterion that globally encompasses the entire arterial tree, and therefore promises better results than local methods.

Though we have assumed a parallel geometry in Section III, the criterion (9) could apply to any geometry, with a different projection function $s\left(\mu_{\psi}\right)$. However, the particular algorithm for minimizing (9) we describe here relies heavily on the fact that, for a parallel (or cylindrical) imaging geometry, the projections of an elliptical cross-section fall on the same row in each of the projection images. This fact is embodied in (1) and (4), which allows us to rewrite (9) as

$$
\begin{aligned}
& \hat{\boldsymbol{\Psi}}=\arg \min _{K} \min _{z_{1}, \cdots, z_{\kappa} x_{1}(\cdot), \cdots, x_{K}(\cdot)} \min ^{N} \\
& {\left[\sum_{n=1}^{N}\left\|\boldsymbol{y}_{n}-\boldsymbol{s}\left(\boldsymbol{x}_{1}\left(z_{n}\right), \cdots, \boldsymbol{x}_{K}\left(z_{n}\right)\right)\right\|^{2}+\boldsymbol{\alpha}^{\prime} \sum_{k=1}^{K} \int \ddot{\boldsymbol{x}}_{k}^{2}(z) d z\right]}
\end{aligned}
$$

where [cf. (4)] the 2-D projection of an ellipse collection is given by

$$
\boldsymbol{s}\left(\boldsymbol{x}_{1}\left(z_{n}\right), \cdots, \boldsymbol{x}_{K}\left(z_{n}\right)\right)=\boldsymbol{s}_{n}\left(\mu_{\left\{\varepsilon\left(x_{1}\left(z_{n}\right)\right), \cdots, \varepsilon\left(x_{K}\left(z_{n}\right)\right\}\right\}}\right) .
$$

By standard arguments [50], [51], based on the Euler equations for the functional (10), the infimum of $\Phi$ is achieved, and any object-set that achieves that infimum is composed of objects whose component functions are cubic-splines with knots at some subset of $z_{1}, \cdots, z_{N}$. A cubic-spline function is determined completely by its values at the knots (sample points). We use this fact to simplify the continuous variational problem (10) into a tractable discrete problem. Note that this discretization is a natural consequence of the form of (10). Define the sample points for the $k$ th object by

$$
\begin{aligned}
\mathfrak{N}_{k} & \triangleq\left\{n: z_{n} \in \mathfrak{Z}_{k}, n=1, \cdots, N\right\}, \\
n_{b, k} & =\min \mathfrak{N}_{k}, \quad n_{e, k}=\max \mathfrak{N}_{k},
\end{aligned}
$$

and the samples by

$$
\boldsymbol{X}_{k} \triangleq\left[\boldsymbol{x}_{k}\left(z_{n_{k, k}}\right)^{\prime}, \cdots, \boldsymbol{x}_{k}\left(z_{n_{c, k}}\right)^{\prime}\right]^{\prime}
$$

where $\boldsymbol{X}_{k}$ denotes the parameters of the $k$ th object on those planes within its length. With these definitions, we can rewrite (10) as

$$
\begin{aligned}
& \hat{\boldsymbol{\Psi}}=\arg \min _{K} \min _{\mathfrak{N}_{1}, \cdots, \mathfrak{N}_{K}} \min _{\boldsymbol{X}_{1}, \cdots, \boldsymbol{X}_{K}} \\
& {\left[\sum_{n=1}^{N}\left\|\boldsymbol{y}_{n}-s\left(\boldsymbol{x}_{1}\left(z_{n}\right), \cdots, \boldsymbol{x}_{K}\left(z_{n}\right)\right)\right\|^{2}+\sum_{k=1}^{K} \boldsymbol{X}_{k}^{\prime} \boldsymbol{S}_{k} \boldsymbol{X}_{k}\right] .}
\end{aligned}
$$

The matrix $S_{k}$, defined in [48, eq. (23)], depends on $\mathfrak{\Re}_{k}$ and $\alpha$, and serves to discretize the integral in (10). 
Though many desirable properties of spline smoothers are known [43], [45], the nonlinearity of (12) limits how much we can say about its theoretical properties. There are probably local minima, and even the global minimum is not unique in general, due to the nonuniqueness discussed in Section IV. However, regularization methods have shown promise in other applications [46], and the empirical results of Section VII likewise are encouraging.

We have defined an optimality criterion for the object reconstruction problem. This criterion can be used to compare suboptimal algorithms, or minimized to generate an arterial tree estimate. In the next section, we present an algorithm for such minimizations.

\section{Estimation Algorithm}

Having defined optimality criterion (12), the object estimation problem becomes simply the problem of designing an algorithm that can compute $\hat{\boldsymbol{\Psi}}$ with a reasonable computational load. A general nonlinear minimization technique would be completely impractical, since there are thousands of parameters to estimate. Fortunately, we can exploit the sparseness of arterial trees and the special matrix structures of vector-spline smoothing to tailor an algorithm to this problem.

The outer two minimizations in (12) can be thought of as a detection operation: estimating the number of arteries and their endpoints. In the remainder of this paper, we focus on the innermost minimization: the problem of estimating the objects' cross-section parameters given the number of objects and an initial estimate.

An initial estimate could come from the output of any of the suboptimal 3-D reconstruction schemes, but we currently use manual entry. A trained operator determines the number of objects, and then enters coarse centerlines using a technique similar to [52]. After tracing a coarse piecewise-linear approximation of each object's centerline on one view, the operator traces the centerline in the (e.g.) orthogonal view using auxiliary lines, observing the other views to confirm object correspondence. Since there are multiple views, the correspondence problem that confounds two-view reconstruction is alleviated. From cubic-spline interpolants of the 2-D centerlines, an initial 3-D skeleton is generated automatically by analytical back-projection. A typical arterial radius for the anatomy of interest is used as the radius for an initially circular cross-section. The result of this procedure is a crude estimate of $\boldsymbol{\Psi}$ that initializes the iterative algorithm presented below.

If the objects were disjoint, and if their projections were overlap free, then the minimization of (12) would decompose into $K$ independent minimizations - one for each object. This fact, combined with the sparseness of arterial trees, suggests that the alternating minimization (AM) algorithm [23], [53] is appropriate for this problem. Here, we use the AM algorithm on an object by object ${ }^{7}$ basis: the parameters of each object are estimated in turn, while holding the other objects fixed, and the procedure is iterated. For disjoint objects, convergence would occur in one iteration, but in general the number of iterations depends on the degree of overlap. That describes the "outer loop" of the algorithm; we now focus on the algorithm for estimating the parameters of a particular object, holding the other objects fixed.

${ }^{7}$ In [23], the AM algorithm was applied on an ellipse by ellipse basis, and the sparseness argument was weaker.
Suppose we are considering the $k$ th object, and $\boldsymbol{X}_{j}$ denote the current parameter estimates of the $j$ th object. Then from the inner minimization of (12):

$$
\begin{array}{r}
\hat{\boldsymbol{X}}_{k}=\arg \min _{\boldsymbol{X}_{k}}\left[\sum_{n=n_{h, k}}^{n_{k, k}} \| \boldsymbol{y}_{n}-\boldsymbol{s}\left(\hat{\boldsymbol{x}}_{1}\left(z_{n}\right), \cdots, \hat{\boldsymbol{x}}_{k-1}\left(z_{n}\right),\right.\right. \\
\left.\left.\boldsymbol{x}_{k}\left(z_{n}\right), \hat{\boldsymbol{x}}_{k+1}\left(z_{n}\right), \cdots, \hat{\boldsymbol{x}}_{K}\left(z_{n}\right)\right) \|^{2}+\boldsymbol{X}_{k}^{\prime} \boldsymbol{S}_{k} \boldsymbol{X}_{k}\right] \\
=\arg \min _{\boldsymbol{X}_{k}}\left[\sum_{n=n_{b, k}}^{n_{e, k}}\left\|\boldsymbol{y}_{n}^{*}-\boldsymbol{s}_{n}^{*}\left(\boldsymbol{x}_{k}\left(z_{n}\right)\right)\right\|^{2}+\boldsymbol{X}_{k}^{\prime} \boldsymbol{S}_{k} \boldsymbol{X}_{k}\right]
\end{array}
$$

where

$$
\boldsymbol{y}_{n}^{*} \triangleq \boldsymbol{y}_{n}-\boldsymbol{s}\left(\hat{\boldsymbol{x}}_{1}\left(z_{n}\right), \cdots, \hat{\boldsymbol{x}}_{j-1}\left(z_{n}\right), \hat{\boldsymbol{x}}_{j+1}\left(z_{n}\right), \cdots, \hat{\boldsymbol{x}}_{K}\left(z_{n}\right)\right) \text {, }
$$$$
\boldsymbol{X}_{k} \triangleq\left[\boldsymbol{x}_{k}\left(z_{n_{b, k}}\right)^{\prime}, \cdots, \boldsymbol{x}_{k}\left(z_{n_{e, k}}\right)^{\prime}\right]^{\prime} \text {, }
$$

and

$$
\begin{aligned}
s_{n}^{*}(x) \triangleq & s\left(x_{1}\left(z_{n}\right), \cdots, \hat{x}_{j-1}\left(z_{n}\right), \boldsymbol{x}, \hat{\boldsymbol{x}}_{j+1}\left(z_{n}\right), \cdots, \hat{\boldsymbol{x}}_{K}\left(z_{n}\right)\right) \\
& -\boldsymbol{s}\left(\boldsymbol{x}_{1}\left(z_{n}\right), \cdots, \hat{\boldsymbol{x}}_{j-1}\left(z_{n}\right), \hat{\boldsymbol{x}}_{j+1}\left(z_{n}\right), \cdots, \hat{\boldsymbol{x}}_{K}\left(z_{n}\right)\right) .
\end{aligned}
$$

$\boldsymbol{y}_{n}^{*}$ is the difference between the measurements and the projections of the fixed objects. By our restriction to bifurcations, the $k$ th ellipse on slice $n$ is either isolated, or it intersects one other ellipse, with index $k_{n}$, say. Thus,

$$
s_{n}^{*}(x)= \begin{cases}s(x), & x_{k} \text { isolated } \\ s\left(x, x_{k_{n}}\right)-s\left(x_{k_{n}}\right), & x_{k} \text { and } x_{k_{n}} \text { intersect }\end{cases}
$$

where $s\left(\boldsymbol{x}, \boldsymbol{x}_{k_{n}}\right)$ is defined by (11). Formulas for $s_{n}^{*}(\boldsymbol{x})$ are given in Appendix B. Note that if the objects were disjoint then $s_{n}^{*}(x)$ and $s(x)$ would be equivalent.

We have finally reduced the original optimization problem (12) down to the form (13), which has been solved previously. In [48], [49], Fessler presented an algorithm for smoothing nonlinear measurements of vector-valued functions that is directly applicable to (13). The computational requirement of that algorithm is only $O(N)$, due to the band matrix structure of spline smoothing. Source code is available through NETLIB [54]. That algorithm uses the nonlinear pseudomeasurement function $s_{n}^{*}(x)$ and its derivatives.

One could use the cross-validation method described in [48], [49] to select the smoothing parameter $\alpha$ automatically in several different ways for this problem. A computationally efficient alternative to cross-validating the entire object-set is the following approach. First, estimate the objects using an educated guess for the smoothing parameter. Then, subtract the projections of all of the objects except one (e.g., the longest) from the measurements, leaving (approximately) only the selected object's contribution. Next, apply the cross-validation method of [49] to choose $\alpha$ automatically for that single-object data set. Finally, estimate the entire object-set using the smoothing factor chosen by cross-validation. The examples of Section VII were based on this approach. Unfortunately, the cross-validation score may have local minima, and a descent from the initial $\boldsymbol{\alpha}$ may not yield the truly optimal $\alpha$. A global search for $\alpha$ on a patient-by-patient basis would be too time consuming; a more 
practical approach would be to search globally for the best $\alpha$ on a training set of images, and then to apply that value of $\alpha$ to subsequent patient studies.

The essence of the estimation algorithm is described by (12), and more detail on the implementation can be found in [30]. The result of this estimation algorithm is a set of parametrically described SGC objects that are converted to GC parameters using Appendix $\mathrm{C}$. One can use these parameters in several ways:

- generate 3-D shaded surface displays directly,

- generate synthetic projections at any angle,

- plot cross-sections, and

- graph parameters (especially radius) versus arterial axis to examine percent stenosis.

Each of these uses is demonstrated in the next section. A bonus for this parametric method is that shaded surface displays are particularly easy to generate since the estimated ellipses are essentially a surface description.

\section{ExPERIMENTAL Results}

We applied the algorithm described above to three data sets: simulated projection images, MR projections of a branching flow phantom, and MR angiograms of a healthy volunteer's carotid arteries. The SNR per view, defined by $2 \rho h_{p} r_{p} / \sigma$, and the important imaging parameters for these case studies are summarized in Table I.

\section{A. Simulated Projections}

Fig. 7 shows four noisy projection images of a simulated arterial tree. These images were generated using the projection model of Section III. Table II displays the convergence of the algorithm for the smoothing parameter chosen by cross-validating the longest object. Each iteration through all five objects required about $35 \mathrm{~s}$ on a SUN Sparcserver. As expected, the first few iterations improve the estimates considerably, with little further improvement after the fourth iteration. The rms errors for ellipse orientation $\phi$ are misleadingly large; many of the cross-sections are very close to circular, in which case the orientation is irrelevant.

A more meaningful evaluation of the shape estimates can be made from Figs. 8-10. (The estimates are taken from the fourth iteration hereafter.) Fig. 8 compares the true and the estimated cross-sections of the main branch over the stenotic portion. The reconstruction has estimated this eccentric stenosis accurately. Similarly, Figs. 9 and 10 compare the true and the estimated cross-sections of two bifurcations. The overlapping ellipses with very different shapes are estimated accurately.

For a more quantitative comparison, Fig. 11 compares the true and the estimated radii (both in SGC parameters) for this example, and shows the presence of a stenosis quite clearly. These parameters are translated into GC parameters using the formulas of Appendix $\mathrm{C}$, and Fig. 12 displays the resulting radius estimation errors for the five objects. The larger radius "errors" at the endpoints of some of the objects are artifacts that are explained by the object-set ambiguity discussed in Section IV: when one ellipse is almost completely inside another ellipse, it contributes very little to the cross-section. Figs. 9 and 10 show that the cross-sections were in fact estimated accurately.

Graphs such as Fig. 11 are useful for computing percent stenosis, but morphology is best viewed through an interactive 3-D display with cut-planes. Fig. 13 shows a shaded surface display
TABLE I

Imaging Parameters for the Data Sets Used to Evaluate the RECONSTRUCTION METHOD

\begin{tabular}{lccc}
\hline & Simulation & MR Phantom & MR Carotid \\
\hline$K$ & 5 & 2 & 4 \\
$W$ & 256 & 160 & 160 \\
$N$ & 256 & 128 & 128 \\
$\Delta_{h}[\mathrm{~mm}]$ & 1 & 0.478 & 0.7 \\
$\Delta_{r}[\mathrm{~mm}]$ & 1 & 0.478 & 0.35 \\
$P$ & 4 & 4 & 4 \\
$\sigma^{2}$ & 3 & 0.0025 & 0.015 \\
SNR & $2.8-6.7$ & $6.3-40.6$ & $2.8-20.2$ \\
$\theta_{1}, \cdots, \theta_{P}$ & $0,45,90,135$ & $22,67,112,157$ & $0,45,108,143$ \\
\hline
\end{tabular}
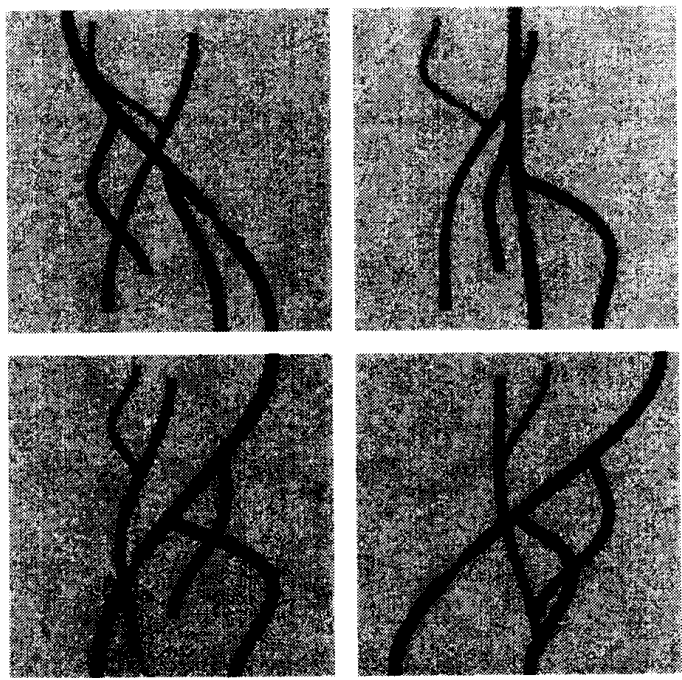

Fig. 7. Simulated angiograms; $\theta=\theta^{\circ}, 45^{\circ}, 90^{\circ}$, and $135^{\circ}$.

TABLE II

rms Parameter Estimation Errors for Eight Iterations

\begin{tabular}{cccllclc}
\hline & \multicolumn{7}{c}{ rms Error } \\
\cline { 2 - 7 } Iteration & \multicolumn{1}{c}{$c_{x}$} & \multicolumn{1}{c}{$c_{y}$} & \multicolumn{1}{c}{$r$} & \multicolumn{1}{c}{$\lambda$} & \multicolumn{1}{c}{$\phi$} & \multicolumn{1}{c}{$\rho$} \\
\hline 0 & 1.188 & 0.9704 & 1.528 & 0.06325 & 23.05 & 0.2044 \\
1 & 0.2884 & 0.2345 & 0.1943 & 0.1352 & 38.25 & 0.03376 \\
2 & 0.1859 & 0.1568 & 0.136 & 0.07881 & 34.66 & 0.01879 \\
3 & 0.1783 & 0.1386 & 0.1133 & 0.06639 & 34.95 & 0.0145 \\
4 & 0.1606 & 0.1174 & 0.1048 & 0.07071 & 34.94 & 0.01396 \\
5 & 0.1573 & 0.1312 & 0.104 & 0.06435 & 34.61 & 0.01451 \\
6 & 0.1456 & 0.1365 & 0.1081 & 0.06774 & 35.2 & 0.0138 \\
7 & 0.1556 & 0.1363 & 0.1055 & 0.067 & 35.05 & 0.01354 \\
8 & 0.1507 & 0.1376 & 0.1031 & 0.06741 & 36.76 & 0.01365 \\
\hline
\end{tabular}

of the reconstruction, generated using the simplest aspects of the shading method presented in [55].

The results from this data set demonstrate the potential performance of object-based reconstruction, and highlight an important advantage of the parametric approach: despite the low SNR, the estimates are of subpixel accuracy. Discrete voxel representations of $\mu$, on the other hand, are typically limited by the measurement resolution. 


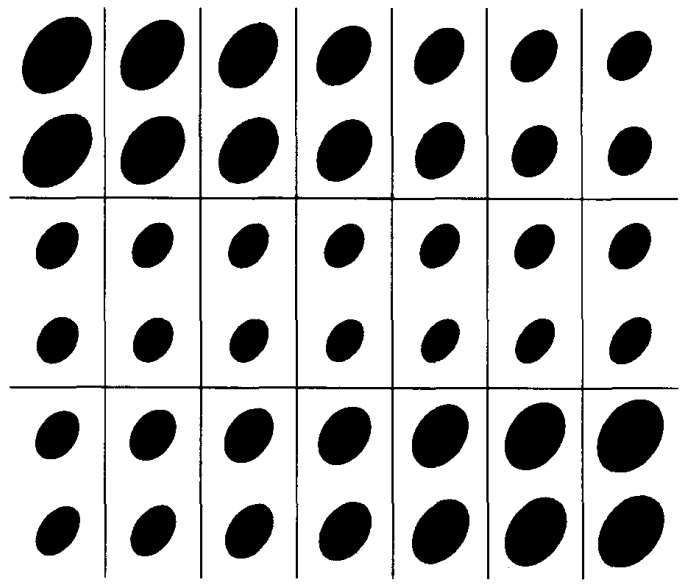

Fig. 8. True (above) versus estimated (below) stenosis cross-sections, $n=160, \cdots, 180$

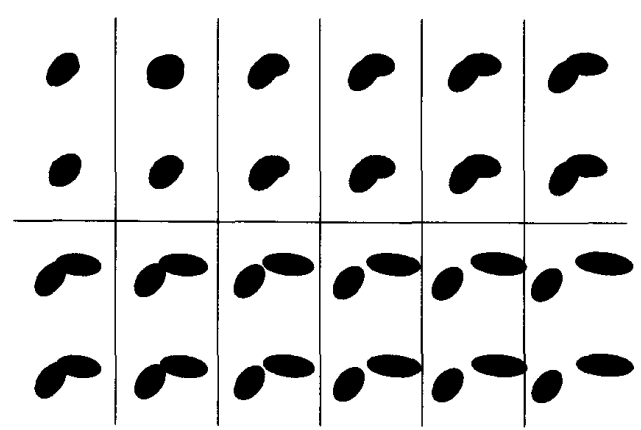

Fig. 9. True (above) versus estimated (below) bifurcation cross-sections: $n=130, \cdots, 141$.

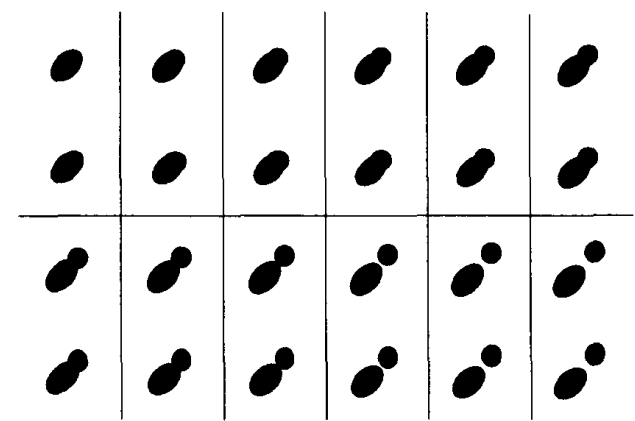

Fig. 10. True (above) versus estimated (below) bifurcation cross-sections $n=80, \cdots, 94$.

\section{B. MR Branching Phantom}

The images in Fig. 14 are MR projections of a $\mathrm{MnCl}$ solution flowing through a plastic $Y$-shaped connector attached to Tygon tubing. We used the projection-reconstruction selective inversion-recovery (PRSIR) [56], [57] method to collect the data. The inner diameter of the $Y$ connector was $3.75 \mathrm{~mm}$. Fig. 15 displays a histogram of the radius estimation error over the $Y$ section. The rms error in radius was only $0.04 \mathrm{~mm}$. As a verification of the geometric consistency of the estimates, Fig. 16
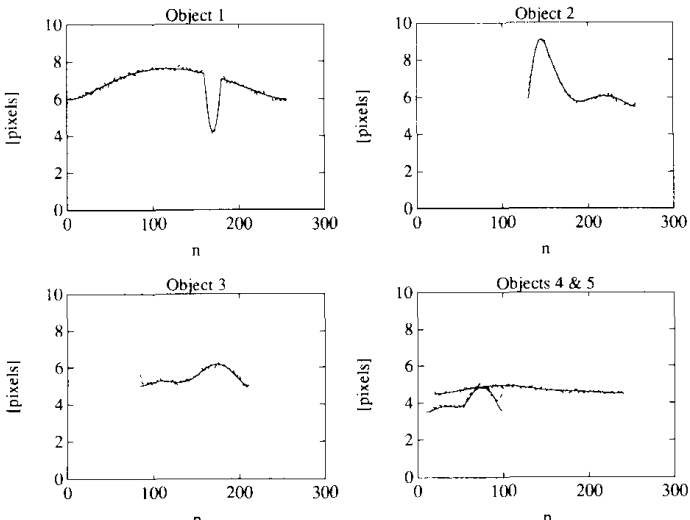

Fig. 11. True (solid) versus estimated (dashed) object radii.
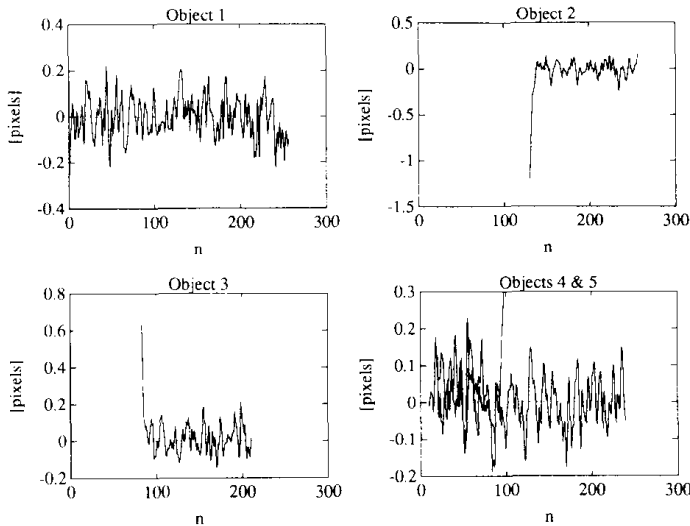

Fig. 12. Radius estimation errors for the simulated objects

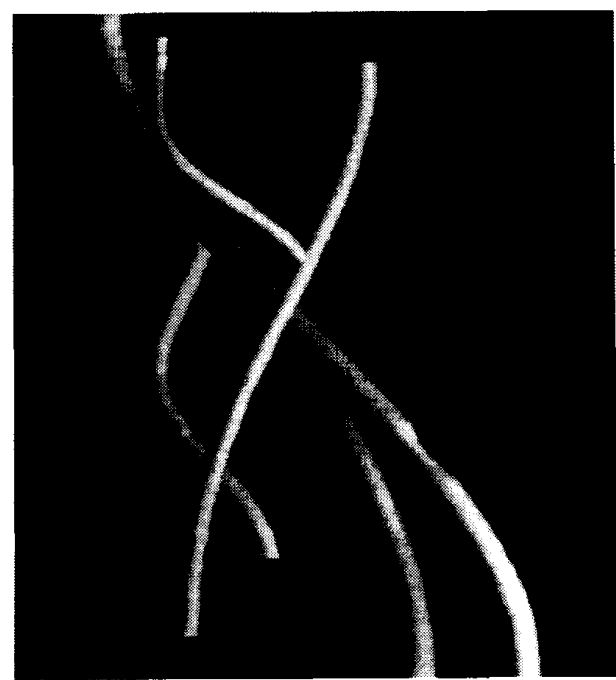

Fig. 13. 3-D surface display of reconstructed simulated arteries.

displays the outline of the projection of the estimates superimposed on another view that was also acquired, but was not used for the reconstruction. The overall correspondence is quite good, 

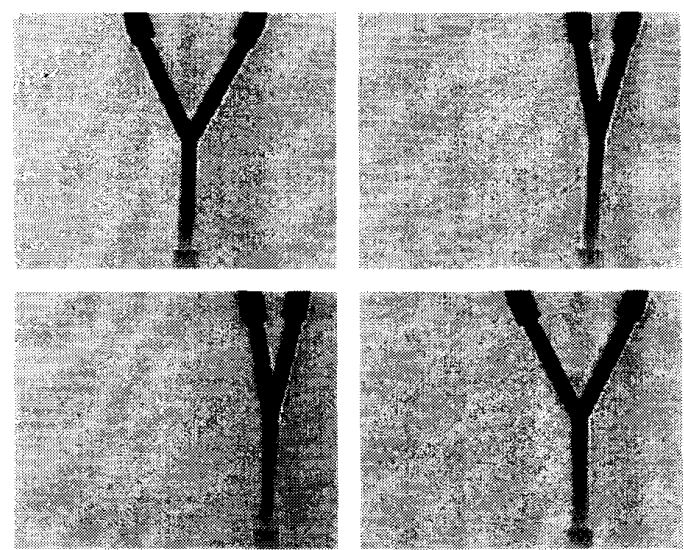

Fig. 14. MR tube-phantom projections: $\theta=22^{\circ}, 67^{\circ}, 112^{\circ}$, and $157^{\circ}$.

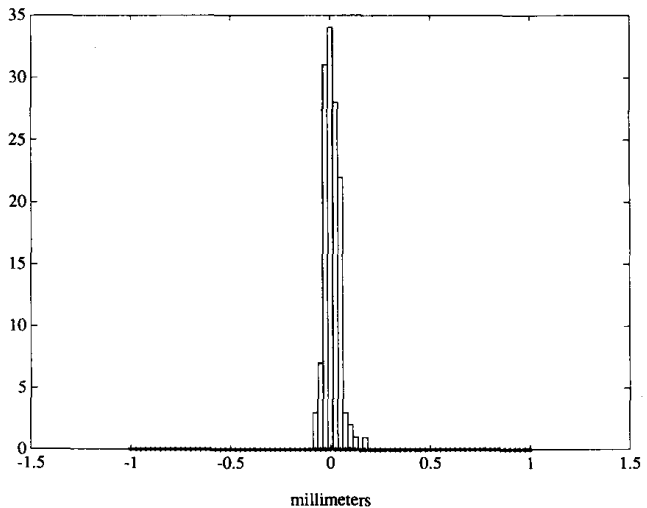

Fig. 15. Histogram of radius estimation error for MR phantom

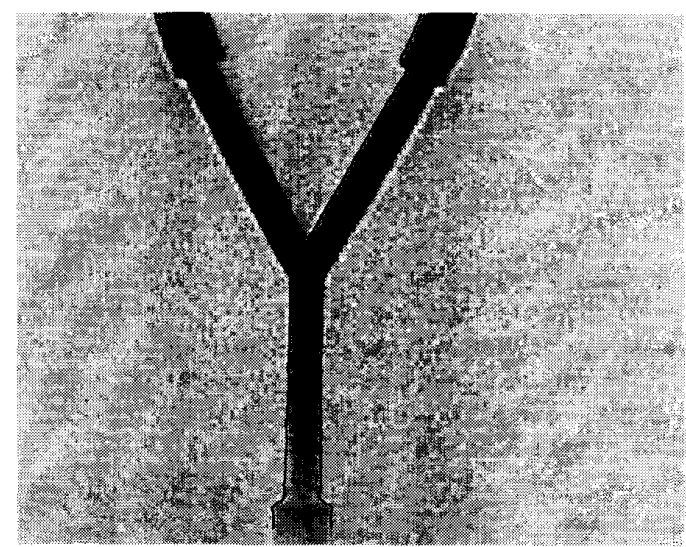

Fig. 16. Reprojection of estimates (solid line) on an unused view; $\theta=0^{\circ}$.

though the sharp transitions between the small branch and the larger tubes would be better modeled by a GC than a SGC, since some of the image rows intersect both the small tube and a corner of the larger tube.
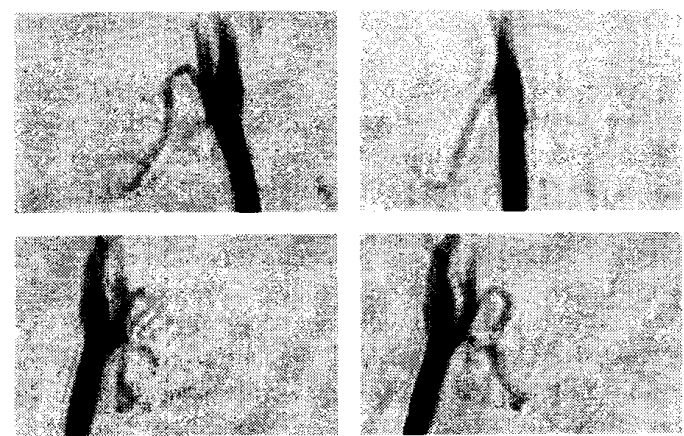

Fig. 17. MR in vivo carotid angiograms; $\theta=0^{\circ}, 45^{\circ}, 108^{\circ}$, and $143^{\circ}$

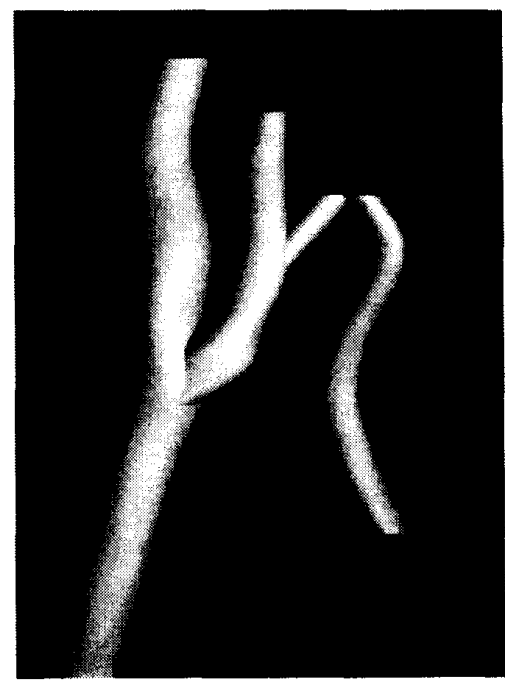

Fig. 18. 3-D surface display of reconstructed carotid arteries.

\section{MR Carotid Angiograms}

Fig. 17 shows four MRSIR projections of a slab containing a healthy volunteer's right carotid artery. A surface coil was placed on the right side of the subject's neck, to maximize SNR. We have no means of making a quantitative evaluation of the results, but Fig. 18 shows a 3-D display of the estimated arteries. This example clearly motivates extending this work beyond single-valued objects, since this facial artery is clearly a multivalued function of $z$.

\section{Discussion}

We have described an object-based method for reconstructing arterial trees from a few projections. By exploiting a priori knowledge of arterial shape and smoothness, subpixel accuracy reconstructions are achieved from only four noisy projection images. The promise of this method is perhaps best demonstrated by Figs. 9 and 10, which show that accurate reconstruction of bifurcations is achievable with parametric models. Note that attempting to reconstruct intersecting ellipses on a local, slice-by-slice basis would be very sensitive to noise; it is the a priori knowledge of smoothness that makes this global approach effective. A possible disadvantage of this approach is 
its complexity; our implementation consists of over ten thousand lines of $C$ code.

To make the approach useful clinically, the manual entry should be eliminated, perhaps by using a detection algorithm based on the outer two minimizations of (12). Brute force minimization of (12) would be impractical computationally; one would need to exploit the structure of arterial trees, as done in Section VI. Automating the procedure should be easier in the high-SNR case, as with intra-arterial contrast studies [58]. Clinical utility would certainly be improved if true GC's could be estimated. The ability of MR to acquire projections in arbitrary directions should be useful for addressing this problem.

Our approach has been to avoid enforcing plausible constraints such as equality of the density of overlapping ellipses. Though such constraints could reduce the degrees of freedom and thereby decrease the estimate variance, they could also increase potential modeling error. Nevertheless, if the SNR is very low, it may be necessary to use even more a priori knowledge. The branching-spline method of Silverman [59], originally applied to agricultural data, may be a one useful approach to enforcing tighter constraints between objects that branch.

An open theoretical question is how to best choose the smoothing factor $\alpha$. Should $\alpha$ be the same for every object, or, since smaller arteries tend to be more tortuous, should $\alpha$ scale with object size? Robust methods for choosing $\alpha$ should also be explored [60]. Having allowed intersecting ellipses, we could also combine ellipses to represent other cross-sections, such as crescent shapes [30]. Presently, only X-ray angiography is used routinely, and we are generalizing the algorithm of this paper to apply to cone-beam projections [30].

\section{APPENDIX A}

\section{ProjeCtion OF AN ElLIPSE}

Consider a collection of ellipses in a given plane, some of which may intersect. Since we restrict our model to bifurcations, the collection's projections are the superposition of the projections of those ellipses that are isolated, plus the sum of the projections of the pairs of intersecting ellipses. Thus, we need two types of formulas: projections of a single ellipse, and projections of intersecting ellipses [cf. (16)]. In this appendix and the next, we present expressions for these projections and their partial derivatives. These are needed by the nonlinear vector-spline smoothing algorithm in [48], [49] since it is a gradient-descent optimization method. Specifically, we derive expressions for $s^{*}(x)$, or equivalently, its components $s_{\theta, i}^{*}(x)$.

Consider a single isolated ellipse with parameters $\boldsymbol{x}=\left[c_{x} c_{y}\right.$ $r \lambda \phi \rho]^{\prime}$. Since we consider projections only in the plane of this ellipse, we drop the dependence on $z$ (and hence $v$ and $n$ ). From (1), (7), and (8), the ideal continuous line-integral of an ellipse is

$$
\begin{aligned}
l_{\theta}(u ; \boldsymbol{x}) & =\int \rho 1_{\varepsilon(x)}(s \cos \theta+u \sin \theta, s \sin \theta-u \cos \theta) d s \\
& =2 \rho h_{p} \sqrt{r_{p}^{2}-\left(u-u_{p}\right)^{2}} 1_{\left|u-u_{p}\right| \leq r_{p}}
\end{aligned}
$$

where

$$
\begin{aligned}
& u_{p} \triangleq c_{x} \sin \theta-c_{y} \cos \theta, \\
& r_{p} \triangleq r \cdot \sqrt{a_{+}}, \quad h_{p} \triangleq a_{+}^{-1},
\end{aligned}
$$

and

$$
a_{ \pm}=\frac{1}{2}\left(\left(\lambda \pm \lambda^{-1}\right)-\left(\lambda \mp \lambda^{-1}\right) \cos (2(\phi-\theta))\right) .
$$

Then from (2) and (3),

$$
s_{\theta, i}(\boldsymbol{x})=h_{i} * l_{\theta, i}(\boldsymbol{x})
$$

where

$$
\begin{aligned}
l_{\theta . i}(\boldsymbol{x}) & =\int_{\left(i-i_{h}-1\right) \Delta h}^{\left(i-i_{h}\right) \Delta h} l_{\theta}(u ; \boldsymbol{x}) d u \\
& =\left.\rho h_{\rho}\left[u \sqrt{r_{p}^{2}-\left(u-u_{p}\right)^{2}}+r_{p}^{2} \arcsin \left(\frac{u}{r_{p}}\right)\right]\right|_{u=u I} ^{u=u_{R}}
\end{aligned}
$$

where

$$
\begin{aligned}
& u_{L} \triangleq \max \left\{\frac{\left(i-i_{h}-1\right) \Delta_{h}}{r_{p}},-1\right\}, \\
& u_{R} \triangleq \min \left\{\frac{\left(i-i_{h}\right) \Delta_{h}}{r_{p}}, 1\right\},
\end{aligned}
$$

for $i$ in the range

$$
\left\{i:\left|\left(i-i_{h}-\frac{1}{2}\right) \Delta_{h}-u_{p}\right| \leq r_{p}+\frac{1}{2} \Delta_{h}\right\},
$$

otherwise the values are 0 . By the linearity of convolution, the partial derivatives of $s_{\theta, i}(\boldsymbol{x})$ are the blurred partial derivatives of $l_{\theta, i}(x)$, which are given below for $i$ in the same range:

$$
\begin{aligned}
& \frac{\partial l_{\theta, i}(\boldsymbol{x})}{\partial c_{x}}=-(\sin \theta)\left(l_{\theta}\left(u_{R} ; \boldsymbol{x}\right)-l_{\theta}\left(u_{L} ; \boldsymbol{x}\right)\right) \\
& \frac{\partial l_{\theta, i}(\boldsymbol{x})}{\partial c_{y}}=(\cos \theta)\left(l_{\theta}\left(u_{R} ; \boldsymbol{x}\right)-l_{\theta}\left(u_{L} ; \boldsymbol{x}\right)\right) \\
& \frac{\partial l_{\theta, i}(\boldsymbol{x})}{\partial r}=2 \rho r\left(\arcsin \left(\frac{u_{R}}{r_{p}}\right)-\arcsin \left(\frac{u_{L}}{r_{p}}\right)\right) \\
& \frac{\partial l_{\theta, i}(\boldsymbol{x})}{\partial \lambda}=\rho \frac{-a_{-}}{\lambda a_{+}^{2}}\left(\sqrt{r_{p}^{2}-\left(u_{R}-u_{p}\right)^{2}}-\sqrt{r_{p}^{2}-\left(u_{L}-u_{p}\right)^{2}}\right) \\
& \frac{\partial l_{\theta, i}(\boldsymbol{x})}{\partial \phi}=\rho \frac{-\left(\lambda-\lambda^{-1}\right) \sin (2(\phi-\theta))}{a_{+}^{2}} \\
& \quad \cdot\left(\sqrt{r_{p}^{2}-\left(u_{R}-u_{p}\right)^{2}}-\sqrt{r_{p}^{2}-\left(u_{L}-u_{p}\right)^{2}}\right) \\
& \frac{\partial l_{\theta, i}(\boldsymbol{x})}{\partial \rho}=l_{\theta, i}(\boldsymbol{x}) / \rho .
\end{aligned}
$$

$s\left(\mu_{\Psi}\right)$ could be implemented as a subroutine that accepts an object-set as its argument and returns a vector of length $N P W$. However, we achieve substantial memory and computational savings by exploiting the fact that projections of ellipses are semi-ellipse "bump" functions whose supports are only small fractions of the size of the projection vector.

\section{APPENDIX B}

Projection of INTERSECTING ElLIPSES

The projection of two intersecting ellipses is more complicated due to the region of overlap. Consider two intersecting

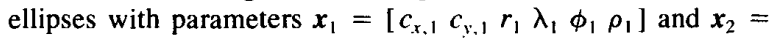

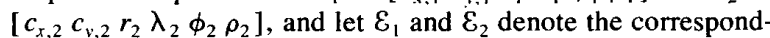
ing ellipse sets (7). From (16), it suffices to derive an expression for $s^{*}\left(x_{1}\right)$, or equivalently its components $s_{\theta, i}^{*}\left(\boldsymbol{x}_{1}\right)$ where 


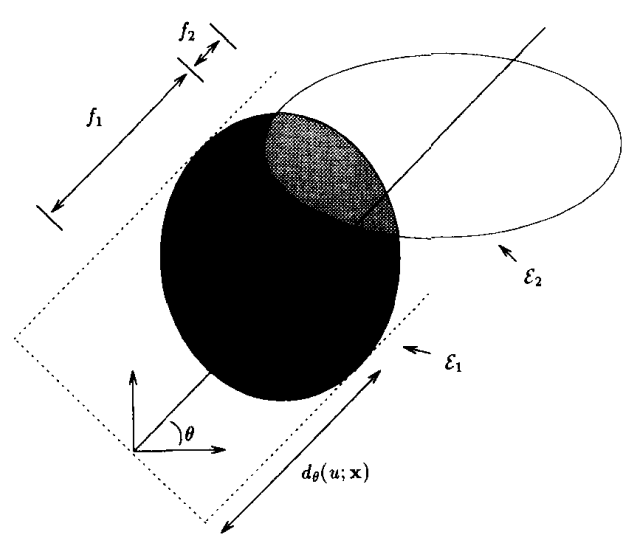

Fig. 19. Line integral through overlapping ellipses.

$\boldsymbol{x}_{2}$ is held fixed. Combining (2), (3), and (16),

$$
s_{\theta, i}^{*}\left(x_{1}\right)=h_{i} * l_{\theta, i}^{*}\left(x_{1}\right)
$$

where

$$
l_{\theta . i}^{*}\left(x_{1}\right) \triangleq \int_{\left(i-i_{h}-1\right) \Delta_{h}}^{\left(i-i_{h}\right) \Delta_{h}} l_{\theta}^{*}\left(u ; x_{1}\right) d u
$$

and

$$
l_{\theta}^{*}\left(u ; x_{1}\right) \triangleq l_{\theta}\left(u ; x_{1}, x_{2}\right)-l_{\theta}\left(u ; x_{2}\right) .
$$

Applying (1) and (8) and using an overline to represent set complements

$$
\begin{aligned}
l_{\theta}^{*}\left(u ; x_{1}\right)= & \int_{\{(x, y): x \sin \theta-y \cos \theta=u\}}\left[\left(\rho_{1} 1_{\varepsilon_{1}}+\rho_{2} 1_{\varepsilon_{2}}\right.\right. \\
& \left.\left.-\frac{\rho_{1}+\rho_{2}}{2} 1_{\varepsilon_{1} \cap \varepsilon_{2}}\right)-\left(\rho_{2} 1_{\varepsilon_{2}}\right)\right] d l \\
= & \int_{\{(x, y): x \sin \theta-y \cos \theta=u\}}\left[\rho_{1} 1_{\mathcal{E}_{1}} \bar{\varepsilon}_{\overline{\varepsilon_{2}}}+\frac{\rho_{1}-\rho_{2}}{2} 1_{\varepsilon_{1}} 1_{\varepsilon_{2}}\right] d l \\
= & \left(\rho_{1}\right) f_{1}\left(d_{\theta}\left(u ; x_{1}\right), l_{\theta}\left(u ; x_{1}\right) / \rho_{1}, d_{\theta}\left(u ; x_{2}\right), l_{\theta}\left(u ; x_{2}\right) / \rho_{2}\right) \\
& +\left(\frac{\rho_{1}-\rho_{2}}{2}\right) f_{2}\left(d_{\theta}\left(u ; x_{1}\right), l_{\theta}\left(u ; x_{1}\right) / \rho_{1}, d_{\theta}\left(u ; x_{2}\right), l_{\theta}\left(u ; x_{2}\right) / \rho_{2}\right),
\end{aligned}
$$

where for $j=1,2$ : $d_{\theta}\left(u ; \boldsymbol{x}_{j}\right) \triangleq c_{x, j} \cos \theta+c_{y, j} \sin \theta$

$$
-\left(u-u_{p, j}\right)\left(\lambda_{j}-\lambda_{j}^{-1}\right) \frac{1}{2 a_{+, j}} \sin \left(2\left(\phi_{j}-\theta\right)\right)
$$

is the distance shown in Fig. 3. The path length functions shown in Fig. 19 are

$$
\begin{aligned}
& f_{1}\left(d_{1}, l_{1}, d_{2}, l_{2}\right) \triangleq \\
& \text { length }\left(\left[d_{1}-l_{1} / 2, d_{1}+l_{1} / 2\right]\right. \\
& \left.\cap \overline{\left[d_{2}-l_{2} / 2, d_{2}+l_{2} / 2\right]}\right), \\
& f_{2}\left(d_{1}, l_{1}, d_{2}, l_{2}\right) \triangleq \\
& \text { length }\left(\left[d_{1}-l_{1} / 2, d_{1}+l_{1} / 2\right]\right. \\
& \left.\cap\left[d_{2}-l_{2} / 2, d_{2}+l_{2} / 2\right]\right)
\end{aligned}
$$

where

length $([a, b] \cap \overline{[c, d]})$

$$
\triangleq \begin{cases}(b-a)-(d-c), & a \leq c, b \geq d \\ b-d, & a \geq c, b \geq d, a \leq d \\ c-a, & a \leq c, b \leq d, b \geq c \\ 0, & \text { otherwise }\end{cases}
$$

length $([a, b] \cap[c, d])$

$$
\triangleq\left\{\begin{array}{ll}
d-c, & a \leq c, b \geq d \\
d-a, & a \geq c, b \geq d, a \leq d \\
b-c, & a \leq c, b \leq d, b \geq c . \\
b-a, & a \geq c, b \leq d \\
0, & \text { otherwise }
\end{array} .\right.
$$

Our implementation uses the following approximation, obtained by interchanging the order of the convolution and the "length" $(\min / \max )$ operations:

$l_{\theta, i}^{*}\left(x_{1}\right) \approx\left(\rho_{1}\right) f_{1}\left(d_{\theta, i}\left(x_{1}\right), l_{\theta, i}\left(x_{1}\right) / \rho_{1}, d_{\theta, i}\left(x_{2}\right), l_{\theta, i}\left(x_{2}\right) / \rho_{2}\right)$

$+\left(\frac{\rho_{1}-\rho_{2}}{2}\right) f_{2}\left(d_{\theta, i}\left(x_{1}\right), l_{\theta, i}\left(x_{1}\right) / \rho_{1}, d_{\theta, i}\left(x_{2}\right), l_{\theta, i}\left(x_{2}\right) / \rho_{2}\right)$ where

$$
d_{\theta . i}(\boldsymbol{x})=d_{\theta}\left(\left(i-i_{h}-\frac{1}{2}\right) \Delta_{h} ; \boldsymbol{x}\right)
$$

From these formulas, we see that the partial derivatives of $s^{*}(x)$ are combinations of those given for $l_{\theta, i}(x)$ in Appendix $\mathrm{A}$, and those of $d_{\theta, i}(\boldsymbol{x})$, given below:

$$
\begin{aligned}
& \frac{\partial d_{\theta, i}}{\partial c_{x}}=\cos \theta+(\sin \theta)\left(\lambda-\lambda^{-1}\right) \frac{1}{2 a_{+}} \sin (2(\phi-\theta)), \\
& \frac{\partial d_{\theta, i}}{\partial c_{y}}=\sin \theta-(\cos \theta)\left(\lambda-\lambda^{-1}\right) \frac{1}{2 a_{+}} \sin (2(\phi-\theta)), \\
& \frac{\partial d_{\theta, i}}{\partial r}=0
\end{aligned}
$$




$$
\begin{aligned}
& \frac{\partial d_{\theta, i}}{\partial \lambda}=-\left(\left(i-i_{h}-\frac{1}{2}\right) \Delta_{h}-u_{p}\right) \sin (2(\phi-\theta)) /\left(\lambda a_{+}\right), \\
& \frac{\partial d_{\theta, i}}{\partial \phi}=\left(\left(i-i_{h}-\frac{1}{2}\right) \Delta_{h}-u_{p}\right)\left(\lambda-\lambda^{-1}\right) \frac{a_{-}}{a_{+}^{2}}, \\
& \frac{\partial d_{\theta, i}}{\partial \rho}=0 .
\end{aligned}
$$

\section{APPENDIX C}

\section{Converting SGC to GC Parameters}

Our reconstruction algorithm provides estimates of the parameters of the elliptical cross-sections of a SGC, as shown in Fig. 2. However, it is the variations in eccentricity, radius, and area perpendicular to an artery's axis that are important diagnostically. In this appendix, we present equations for converting between the parameters of a GC and a SGC. These relations are derived by approximating a SGC locally by an elliptical cone. The position attribute is identical for the GC and SGC models. To first order, the content attribute is also the same, since density usually varies quite slowly. Suppose the estimated shape parameters are $\left[r_{S} \lambda_{S} \phi_{S}\right]$, and that the local slope of the $\mathrm{SGC}$ is $\beta_{x}$ and $\beta_{v}$. Let $\beta=\sqrt{1+\beta_{x}^{2}+\beta_{y}^{2}}$, then

$$
r=\frac{r_{S}}{\sqrt{\beta}}
$$

and

$$
\begin{aligned}
\lambda+\lambda^{-1}= & \beta^{-1} \lambda_{S}+\beta \lambda_{S}^{-1}+\left(\beta-\beta^{-1}\right) \\
& \cdot\left(\lambda_{S}-\lambda_{S}^{-1}\right) \sin ^{2}\left(\phi_{S}-\arctan \left(\beta_{y} / \beta_{x}\right)\right) .
\end{aligned}
$$

Ellipse orientation is not well defined for an arbitrary GC axis [25] in general.

\section{ACKNOWLEDGMENT}

The authors are grateful for the invaluable assistance of $S$. Wang and D. Nishimura in acquiring the MR data, homodyning software provided by D. Noll, graphics wizardry of C. Barrett, and the helpful comments of the reviewers.

\section{REFERENCES}

[1] J. Ross et al., "Guidelines for coronary angiography," Circulation, vol. 76, pp. 963A-977A, Oct. 1987.

[2] S. E. Epstein, A. A. Quyyumi, and R. O. Bonow, "Sudden cardiac death without warning," New England J. Med., vol. 321, pp. $320-324$, Aug. 1989.

[3] D. G. Nishimura, A. Macovski, J. M. Pauly, and S. M. Conolly, "MR angiography by selective inversion recovery," Magn. Resonance Med., vol. 4, pp. 193-202, 1987.

[4] D. G. Nishimura, A. Macovski, J. I. Jackson, R. S. Hu, C. A Stevick, and L. Axel, "Magnetic resonance angiography by selective inversion recovery using a compact gradient echo sequence," Magn. Resonance Med., vol. 8, pp. 96-103, 1988

[5] S. Wang, D. G. Nishimura, and A. Macovski, "Multiple readout selective inversion recovery angiography," Magn. Resonance Med., vol. 17, p. 224, 1991

[6] C. Dumoulin et al., "Three-dimensional phase contrast angiography," Magn. Resonance Med., vol. 9, pp. 139-149, Jan. 1989

[7] T. Masaryk et al. " "3DFT MR angiography of the carotid bifurcation: A comparison of time-of-flight techniques," in Proc. Eighth SMRM, p. 164, Aug. 1989
[8] D. J. Rossi and A. S. Willsky, "Reconstruction from projections based on detection and estimation of objects-Parts I and II: Performance analysis and robustness analysis," IEEE Trans. Acoust., Speech, Signal Processing, vol. 32, pp. 886-906, Aug. 1984.

[9] K. Kitamura, J. M. Tobis, and J. Sklansky, "Estimating the 3-D skeletons and transverse areas of coronary arteries from biplane angiograms," IEEE Trans. Med. Imaging, vol. 7, pp. 173-187, Sept. 1988.

[10] G. Jividen and R. Kasturi, "A survey of image processing techniques for angiograms," vol. TR-88-048, Univ. Park Campus, Penn. State, 1988 .

[11] M. Block, A. A. Bove, and E. L. Ritman, "Coronary angiographic examination with the dynamic spatial reconstructor," Circulation, vol. 70, pp. 209-216, Aug. 1984.

[12] W. E. Higgins, W. J. T. Spyra, and E. L. Ritman, "Automatic extraction of the arterial tree from 3-D angiograms," in Proc. 1lth Annu. Int. Conf. IEEE Eng. Med. Biol. Soc., Nov. 1989, pp. 563-564.

[13] D. L. Parker, D. L. Pope, R. Van Bree, and H. W. Marshall, "Three-dimensional reconstruction of moving arterial beds from digital subtraction angiography," Comput. Biomed. Res., vol. 20, pp. 166-185, 1987

[14] J. Wu, D. L. Parker, G. R. Caputo, and H. W. Marshall, "A clinical feasibility study of $3 \mathrm{D}$ reconstruction and measurement of coronary arteries," Dynamic Cardiovasc. Imaging, vol. 1, no. 4, pp. 273-278, 1988.

[15] M. Siebes, D. Z. D'Argenio, and R. H. Selzer. "Computer assessment of hemodynamic severity of coronary artery stenosis from angiograms," Comput. Methods Programs Biomed., vol. 21 , pp. 143-152, 1985

[16] A. Rougee, K. M. Hanson, and D. Saint-Felix, "Comparison of 3-D tomographic algorithms for vascular reconstruction." in Proc. SPIE 914, Med. Imaging II: Image Form., Detect., Processing, Interpret., 1988, pp. 397-405.

[17] Y. Trousset, D. Saint-Felix, A. Rougée, and C. Chardenon, "Multiscale cone-beam X-ray reconstruction," in Proc. SPIE Med. Imaging IV, Feb. 1990.

[18] K. Shmueli, W. R. Brody, and A. Macovski, "Estimation of blood vessel boundaries in X-ray images," Opt. Eng., vol. 22, pp. 110-116, Jan. 1983.

[19] T. N. Pappas and J. S. Lim, "A new method for estimation of coronary artery dimensions in angiograms," IEEE Trans. Acoust., Speech, Signal Processing, vol. 36, pp. 1501-1513, Sept. 1988.

[20] Y. Bresler and A. Macovski, "Three-dimensional reconstruction from projections with incomplete and noisy data by object estimation," IEEE Trans. Acoust., Speech, Signal Processing, vol. 35, pp. 1139-1152, Aug. 1987.

[21] D. J. Rossi, A. S. Willsky, and D. M. Spielman, "'Object shape estimation from tomographic measurements-A performance evaluation," Signal Processing, vol. 18, pp. 63-88, Sept. 1989.

[22] Y. Bresler, J. A. Fessler, and A. Macovski " Model based estimation techniques for 3-D reconstruction from projections, "Machine Vision Appl., vol. 1, no. 2, pp. 115-126, 1988.

[23] _ , "A Bayesian approach to reconstruction from incomplete projections of a multiple object 3-D domain," IEEE Trans. Pattern Anal. Machine Intell., vol. 11, pp. 840-858, Aug. 1989.

[24] J. R. Spears, T. Sandor, D. S. Baim, and S. Paulin, "The minimum error in estimating coronary luminal cross-sectional area from cineangiographic diameter measurements," Catheter. Cardiovasc. Diagn., vol. 9, pp. 119-128, 1983

[25] Y. Bresler, "Model based estimation techniques for 3-D reconstruction from projections," Ph.D. dissertation, Stanford Univ., Stanford, CA, Dec. 1985

[26] J. J. Gerbrands, G. J. Troost, J. H. C. Reiber, C. J. Kooijman, and C. H. Slump, "3-D reconstruction of coronary arterial segments from two projections," in Proc. 6th IEEE Int. Conf. Pattern Recogn., Munich, Germany, 1982, pp. 270-272.

[27] K. R. Hoffmann, K. Doi, H. Chan, and K. Chua, "Computer reproduction of the vasculature using an automated tracking method," in Proc. SPIE 767, Med. Imaging, 1987, pp. 449-453.

[28] S. R. Fleagle, M. R. Johnson, C. J. Wilbricht, D. J. Skorton, R. F. Wilson, C. W. White, M. L. Marcus, and S. M. Collins, 
"Automated analysis of coronary arterial morphology in cineangiograms: geometric and physiologic validation in humans,' IEEE Trans. Med. Imaging, vol. 8, pp. 387-400, Dec. 1989.

[29] J. A. Fessler and A. Macovski, "3-D reconstruction of vessels with stenoses and aneurysms from dual bi-plane angiograms," in Proc. SPIE 1092, Med. Imaging III: Image Processing, Feb. 1989, pp. 22-32.

[30] J. Fessler, "Object-based 3-D reconstruction of arterial trees from a few projections," Ph.D. dissertation, Stanford Univ., Stanford, CA, Aug. 1990.

[31] A. Macovski, Medical Imaging Systems. Englewood Cliffs, NJ: Prentice-Hall, 1983

[32] A. Macovski and D. C. Noll, "Homodyne detection for improved SNR and partial $k$-space reconstructions," in Book of $A b$ stracts, p. 815, Soc. Magn. Resonance Med., Aug. 1988.

[33] D. C. Noll, D. G. Nishimura, and A. Macovski, "Homodyne detection in magnetic resonance imaging," IEEE Trans. Med. Imaging, to be published.

[34] S. Wang et al., "Ímproved static tissue suppression for selective inversion recovery angiography," 1990 (in preparation)

[35] P. Webb and A. Macovski, "Rapid, fully-automatic, arbitrary volume, in-vivo shimming," Magn. Reson. Med., to be published.

[36] W. A. Edelstein, G. H. Glover, C. J. Hardy, and R. W. Redington, "The intrinsic signal-to-noise ratio in NMR imaging," Magn. Reson. Med., vol. 3, pp. 606-618, 1986

[37] D. P. Giddens, C. K. Zarins, S. Glagov, B. K. Bharadvaj, and D. N. Ku, "Flow and atherogenesis in the human carotid bifurcation," in Fluid Dynamics as a Localizing Factor for Atherosclerosis, G. Schettler et al., Eds. Berlin: Springer-Verlag, 1983 , pp. 38-45.

[38] P. Grottum, A. Svinland, and L. Walløe, "Localization of atherosclerotic lesions in the bifurcations of the main left coronary artery," Atherosclerosis, vol. 47. pp. 55-62, 1983.

[39] T. Binford, "Visual perception by computer," in Proc. IEEE Conf. Syst. Contr., Miami, FL, Dec. 1971.

[40] G. Agin and T. Binford, "Computer description of curved objects," IEEE Trans. Comput., vol. C-25, pp. 439-449, Apr. 1976

[41] D. Marr, Vision. San Francisco, CA: Freeman, 1982.

[42] J. A. Fessler, D. G. Nishimura, and A. Macovski, "Model-based 3-D reconstruction of branching vessels," in Proc. I/th Annu. Int. Conf. IEEE Eng. Med. Biol. Soc,, (Seattle), pp. 561-562, Nov. 1989.

[43] R. L. Eubank, Spline Smoothing and Nonparametric Regression. New York: Dekker; 1988.

[44] M. Zakai and J. Ziv. "On the threshold effect in radar range estimation," IEEE Trans. Inform. Theory, pp. 167-169, Jan. 1969.
[45] B. W. Silverman, "Some aspects of the spline smoothing approach to non-parametric regression curve fitting," J. Royal Statist. Soc. Series $B$, vol. 47 , no. 1, pp. 1-52, 1985.

[46] M. Bertero, T. Poggio, and V. Torre, "Ill posed problems in early vision," Proc. IEEE, vol. 76, pp. 869-899, Aug. 1988

[47] G. Wahba and S. Wold, "A completely automatic French curve: fitting spline functions by cross validation," Commun. Statist., vol. 4, no. 1, pp. 1-17, 1975.

[48] J. A. Fessler, "Non-parametric fixed-interval smoothing with vector splines," IEEE Trans. Acoust., Speech, Signal Processing, to be published.

[49] J. A. Fessler, "Non-parametric fixed-interval smoothing of nonlinear measurements," IEEE Trans. Acoust., Speech, Signal Processing, to be published.

[50] I. J. Schoenberg, "On interpolation by spline functions and its minimal properties," in On Approximation Theory, Proc. Conf., Oberwolfach, Black Forest, pp. 109-129, Aug. 1963.

[51] C. H. Reinsch, "Smoothing by spline functions," Numerische Mathematik, vol. 10, pp. 177-183, 1967.

[52] S. MacKay, R. Sayre, and M. Potel, "3D Galatea: Entry of threedimensional moving points from multiple perspective views," Computer Graph., vol. 16, pp. 213-222, July 1982.

[53] I. Ziskind and M. Wax, "Maximum likelihood localization of multiple sources by alternating projection," IEEE Trans. Acoust., Speech, Signal Processing, vol. 36, pp. 1553-1560, Oct. 1988.

[54] J. J. Dongarra and E. Grosse, "Distribution of mathematical software via electronic mail," Commun. $A C M$, vol. 30, pp. 403407, Oct. 1987.

[55] D. Gordon and R. Reynolds, "Image space shading of 3-dimensional objects," Comput. Vision, Graph., Image Processing, vol. 29, pp. 361-376, 1985.

[56] D. G. Nishimura, J. M. Pauly, and A. Macovski, "MR angiography using projection-reconstruction imaging," in Magn. Resonance Imaging-SMRI 8th Annu. Meet. Program Abstracts, Feb. 1990, p. 107.

[57] D. G. Nishimura, J. I. Jackson, J. M. Pauly, and A. Macovski, "On the nature and reduction of the displacement artifact in flow images," Magn. Reson. Med., to be published.

[58] P. Suetens, P. J. Jansen, A. Haegemans, A. Oosterlinck, and J. Gybels, "3-D reconstruction of the blood vessels of the brain from a stereoscopic pair of subtraction angiograms," Image Vision Comput., vol. 1, pp. 43-51, Feb. 1983.

[59] B. W. Silverman and J. T. Wood, "The non-parametric estimation of branching curves," J. Amer. Statist. Assoc. vol. 82, pp. 551-558, June 1987.

[60] T. Robinson and R. Moyeed, "Making robust the cross-validatory choice of smoothing parameter in spline smoothing regression," Commun. Statist. - Theory Meth., vol. 18, no. 2, pp. 523$539,1989$. 